\title{
Dynamic shaping of transport-reaction processes with a combined sliding mode controller and Luenberger-type dynamic observer design
}

\author{
Davood Babaei Pourkargar and Antonios Armaou ${ }^{1}$ \\ Department of Chemical Engineering, The Pennsylvania State University, University Park, PA 16802
}

\begin{abstract}
We focus on shaping the long-term spatiotemporal dynamics of transport-reaction processes which can be described by semi-linear partial differential equations (PDEs). The dynamic shaping problem is addressed via error dynamics regulation between the governing PDE and a target PDE which describes the desired spatiotemporal behavior. A model order reduction methodology is utilized to construct the required reduced order models (ROMs) for governing and target dynamics via Galerkin's method. We subtract the governing from the target ROMs to obtain reduced offset dynamics error. Then an output feedback sliding mode control structure is synthesized to stabilize the reduced error dynamics and correspondingly synchronize the system and the target spatiotemporal behaviors. A Luenberger-type dynamic observer is applied to estimate the states of the governing ROM required by the sliding mode controller. The proposed approach is applied to address the thermal spatiotemporal dynamic shaping problem in a tubular chemical reactor.
\end{abstract}

Keywords: Dynamic shaping, distributed parameter systems, model order reduction, sliding mode control, dynamic observer, process control

\section{Introduction}

Recently there has been an increasing focus on modeling and control of distributed parameter systems (DPSs) in chemical process and advanced material production industries. Such type of systems frequently arise in a wide range of chemical processes, e.g., fixed and fluidized bed reactors, polymerization and crystallization processes, chemical vapor deposition systems and semiconductor manufacturing processes, due to the existence of diffusion, dispersion and convection mechanisms $[1,21,44,53,62]$. It is imperative to tightly control these processes so that there are zero product quality excursions, even when the process objectives dynamically change which is a usual occurrence in such industrial applications. While DPSs can be mathematically described by partial differential equations (PDEs) and the control problem is a difficult

\footnotetext{
${ }^{1}$ Corresponding author, Tel: +1(814)865-5316, Fax: +1(814)865-7846, Email address: armaou@engr.psu.edu
} 
task due to the spatial distribution of the system states $[17,21,25,42,51,58]$, it becomes even more complicated in the case of chemical DPSs where chemical reactions take place leading to nonlinearities in the governing equation.

Focusing on transport-reaction processes with significant diffusive mechanisms and their mathematical description, we note that they can be described by semi-linear dissipative PDEs whose infinite-dimensional representation in an appropriate functional subspace can be partitioned into two subsystems; slow (and possibly unstable) and fast (and stable), with a time scale dynamic separation [21]. Considering such property, model order reduction (MOR) methodologies have been extensively used in modeling and control of chemical DPSs $[7,10,11,12,16,21,26,28,35]$. Galerkin's method is one of the typical approaches to implement MOR. The required basis functions in such approach can only be computed analytically if and only if the spatial differential operator is linear and the process operates over regular domains. Statistical approaches can be employed as an alternative solution to compute the empirical basis functions of a general class of DPSs from an ensemble of solution profiles. Proper orthogonal decomposition is one of the commonly used statistical techniques to find the optimal set of empirical basis functions for a representative set of solution data which has been widely applied in model reduction, optimization and control of DPSs $[2,5,6,36,55]$ where geometric and Lyapunov based approaches have been used.

Sliding mode control is a variable structure nonlinear control method which changes the nonlinear system dynamics by applying a discontinuous control signal $[41,56]$. The sliding mode controller forces the system dynamics to slide along the boundaries of the system normal behavior called "sliding surface" $[27,63]$. The discontinuous nature of the controller structure causes insensitivity to parameter variations and complete disturbance rejection [13]. Sliding mode optimization and controller designs have been applied in a wide range of chemical, mechanical and electrical systems [14, 15, 30, 35, 50].

To implement the model-reduced controller design for DPSs we need an accurate estimation of the states of the governing reduced order models (ROMs). Static observer designs which were widely employed to estimate such desired states $[21,26,64]$, require the number of measurement sensors that must be supernumerary to the number of ROM states. One of the solutions to circumvent such requirement is applying dynamic observers which theoretically need only one measurement sensor $[4,5,8]$. While 
dynamic observer synthesis has reached an extensive maturity for lumped parameter systems described by ordinary differential equations (ODEs) [33, 37, 38, 39, 40, 49, 59, 61], the synthesis problem remains challenging for DPSs $[24,32,57,65,66]$.

In this paper we consider the spatiotemporal dynamic shaping of transport-reaction processes via MOR. The dynamic shaping problem is addressed by regulating the error dynamics between the governing PDE and a desired spatiotemporal dynamics which are described by a target PDE with the same spatial differential operator. The governing target PDEs are discretized by applying Galerkin's method to obtain ROMs in the form of low-dimensional modal ODEs when the required dominant basis functions are computed analytically by solving the eigenproblem of the linear part of the spatial differential operator. The error dynamics between the governing and target systems is derived by subtracting the ROMs in the form of low-dimensional ODEs which describe the spatiotemporal error dynamics. Then an output feedback control structure is synthesized to stabilize the error dynamics. The control structure is considered as a combination of a Lyapunov-based sliding mode controller $[41,56]$ and a Luenberger-type dynamic observer to estimate the system modes.

The remainder of the paper is organized as follows; a mathematical description of the studied class of semi-linear DPSs and their properties are presented in Section 2. Section 3 presents a short description of the used MOR method. The spatiotemporal dynamic shaping problem is addressed via an output feedback sliding mode control structure synthesis in Section 4. Finally, the proposed dynamic shaping method is successfully illustrated on thermal dynamic shaping inside a tubular chemical reactor described by a semi-linear PDE in Section 5.

\section{Preliminaries}

\subsection{Problem formulation}

To formulate the spatiotemporal dynamic shaping problem we consider a 1D transport-reaction process which can be described by a semi-linear PDE, 


$$
\begin{aligned}
& \frac{\partial}{\partial t} X(z, t)=\mathcal{A}_{n}(z) X(z, t)+F(z, X(z, t))+B(z) u(t), \\
& y(t)=\int_{\Omega} s(z) X(z, t) d z \\
& q\left(X, \frac{\partial X}{\partial z}, \ldots, \frac{\partial^{n-1} X}{\partial z^{n-1}}\right)=0 \text { on } \partial \Omega \\
& X(z, 0)=X_{0}(z)
\end{aligned}
$$

where

$$
\begin{aligned}
& X(z, t) \in \mathbb{R}: \text { spatiotemporal state of the system, } \\
& z \in \Omega: 1 D \text { spatial coordinate, } \\
& t: \text { time, } \\
& \Omega: \text { process domain, } \\
& \partial \Omega: \text { process boundaries, } \\
& \mathcal{A}_{n}(z): \text { linear spatial differential operator of order } n, \\
& F(z, X(z, t)): \text { smooth Lipschitz nonlinear function, } \\
& u(t) \in \mathbb{R}^{l}: \text { vector of manipulated inputs, } \\
& B(z): \text { spatial distribution of manipulated inputs, } \\
& y(t) \in \mathbb{R}^{p}: \text { vector of contentious measurements, } \\
& s(z): \text { vector of measurements' spatial distribution, } \\
& q\left(X, \frac{\partial X}{\partial z}, \ldots, \frac{\partial^{n-1} X}{\partial z^{n-1}}\right): \text { vector of linear homogeneous boundary conditions, } \\
& X_{0}(z): \text { initial spatial profile of the system state. }
\end{aligned}
$$

The dissipative PDE of (1) is linearly dominant, i.e., the spatial differential operator is purely linear and the nonlinearity only appears as a Lipschitz function in the system dynamics. Such equation arises in the majority of transport-reaction processes in the chemical process industries $[21,53]$, where the linear term of $\mathcal{A}_{n}(z) X(z, t)$ indicates the transport (diffusion, dispersion and convection) component and the nonlinear term of $F(z, X(z, t))$ expresses the reaction dynamics.

Remark 1. According to the Lipschitz property of the nonlinear function of $F(z, X(z, t))$ which makes it to be sufficiently smooth, the Picard-Lindelöf theorem can be applied to guarantee the existence and uniqueness of the solution [60]. 


\subsection{System representation}

The studied DPS which is described by the PDE of (1), can be represented in the abstract infinitedimensional form of

$$
\begin{aligned}
& \dot{x}(t)=A x(t)+f(x(t))+b u(t), x(0)=x_{0}, \\
& y(t)=S x(t),
\end{aligned}
$$

by defining the functional state of $x(t) \in \mathbb{W}$,

$$
x(t)=X(\cdot, t)
$$

the linear differential operator,

$$
A x(t)=\mathcal{A}(z) X(\cdot, t)
$$

the nonlinear function,

$$
f(x(t))=F(z, X(\cdot, t))
$$

and the manipulated input operator,

$$
b u(t)=B(z) u(t)
$$

in an appropriate Sobolev subspace of $\mathbb{W}$,

$$
\mathbb{W}(\Omega)=\left\{\mathcal{H}, \frac{\partial \mathcal{H}}{\partial z}, \ldots, \frac{\partial^{n-1} \mathcal{H}}{\partial z^{n-1}} \in \mathcal{L}_{2}(\Omega): q\left(\mathcal{H}, \frac{\partial \mathcal{H}}{\partial z}, \ldots, \frac{\partial^{n-1} \mathcal{H}}{\partial z^{n-1}}\right)=0\right\}
$$

equipped with the following inner product and norm,

$$
\begin{aligned}
& (\mathcal{H}, \mathcal{G})=\int_{\Omega} r(z) \mathcal{H}^{T} \mathcal{G} d z \\
& \|\mathcal{H}\|_{2}=(\mathcal{H}, \mathcal{H})^{1 / 2}
\end{aligned}
$$

where $\mathcal{H}$ and $\mathcal{G}$ are elements of $\mathbb{W}$, and $\mathcal{H}^{T}$ denotes the transpose. Note that the inner product weighting function, $r(z)$, is considered to be 1 to simplify the analysis.

Assumption 1. We assume that the DPS described by the PDE of (1) and its infinite-dimensional representation, (2), is approximately observable and controllable [25]. 


\section{Model order reduction via Galerkin's method}

The infinite-dimensional functional representation of (2) can be projected into an infinite set of ODEs of the system vectorized eigenmodes using standard Galerkin's method. The required eigenfunctions to discretize the states of the Sobolev subspace are the solution of the following eigenproblem,

$$
\begin{aligned}
& A \phi_{i}=\lambda_{i} \phi_{i} \\
& q\left(\phi_{i}, \frac{d \phi_{i}}{d z}, \ldots, \frac{d^{n-1} \phi_{i}}{d z^{n-1}}\right)_{\partial \Omega}=0
\end{aligned}
$$

for $i=1, \ldots, \infty$, where $\lambda_{i}$ and $\phi_{i}$ denote the $i^{\text {th }}$ eigenvalue and its corresponding orthogonal eigenfunction, respectively. Note that the resulting countable set of eigenfunctions is a strong generator of the defined Sobolev subspace, i.e., $\mathbb{W} \triangleq \operatorname{span}\left\{\phi_{i}\right\}_{i=1}^{\infty}$. To apply Galerkin's method we also require defining the adjoint eigenfunctions which satisfy the orthonormal property,

$$
\left(\phi_{i}, \phi_{j}^{*}\right)=\delta_{i j}
$$

where $\phi_{i}^{*} \in \mathbb{W}$ indicates the $i^{\text {th }}$ adjoint eigenfunction and $\delta_{i j}$ the Kronecker delta.

For the majority of transport-reaction processes where diffusion and dispersion play an important role we can assume a time-scale separation in the eigenspectrum of the linear operator of $A$. This assumption is formally stated as:

Assumption 2. Assume the eigenspectrum of linear operator of $A$ denoted by $\Lambda(A) \triangleq\left\{\lambda_{1}, \lambda_{2}, \ldots\right\}$, where $\operatorname{Re}\left(\lambda_{1}\right) \geq \operatorname{Re}\left(\lambda_{2}\right) \geq \cdots \geq \operatorname{Re}\left(\lambda_{m}\right) \geq \operatorname{Re}\left(\lambda_{m+1}\right) \geq \cdots$, satisfies $\operatorname{Re}\left(\lambda_{m+1}\right)<0, \sigma=\frac{\left|\operatorname{Re}\left(\lambda_{1}\right)\right|}{\left|\operatorname{Re}\left(\lambda_{m+1}\right)\right|}$, where $\operatorname{Re}(\cdot)$ denotes the real part and $\sigma$ is a small positive number. According to such separation the eigenspectrum and corresponding Sobolev subspace, $\mathbb{W} \triangleq \operatorname{span}\left\{\phi_{i}\right\}_{i=1}^{\infty}$, can be partitioned into the following subsets and subspaces;

1. finite subset of first $m$ eigenvalues, $\Lambda_{s}(A) \triangleq\left\{\lambda_{1}, \lambda_{2}, \ldots, \lambda_{m}\right\}$, which are slow and possibly unstable, and its corresponding slow Sobolev subspace, $\mathbb{W}_{s} \triangleq \operatorname{span}\left\{\phi_{i}\right\}_{i=1}^{m}$,

2. complement infinite subset of the remainder eigenvalues, $\Lambda_{f}(A) \triangleq\left\{\lambda_{m+1}, \lambda_{m+2}, \ldots\right\}$, which are fast and stable, and its corresponding fast Sobolev subspace, $\mathbb{W}_{f} \triangleq \operatorname{span}\left\{\phi_{i}\right\}_{i=m+1}^{\infty}$,

where $\mathbb{W}=\mathbb{W}_{s} \cup \mathbb{W}_{f}$. 
Parameter $\sigma$ needs to be a small positive number to ensure a proper separation between the finite dimensional slow and possibly unstable, and infinite dimensional fast and stable subsystems. A common value used in transport-reaction processes dynamics area is $\sigma \simeq 0.1$. The condition of small $\sigma$ in Assumption 2 is satisfied by the majority of transport-reaction processes [1, 20, 44, 47, 62]. The small value for $\sigma$ is not generally satisfied for

1. Convection-reaction processes described by first-order hyperbolic PDEs where the eigenvalues cluster along vertical (or nearly vertical), asymptotes in the complex plane (see Chapter 2 in [21] for more details).

2. Diffusion-reaction processes described by parabolic PDEs for which the spatial coordinate is defined in the infinite domain, where wavy behavior is usually exhibited as the result of continuous eigenspectrum (see $[21,48]$ for more details).

In such cases, a modal separation in the system dynamics can be enforced by appropriate controller design $[21,22,23]$.

Taking advantage of Assumption 2, the Galerkin integral projectors can be defined,

$$
\begin{aligned}
& \mathcal{P}: \mathbb{W} \rightarrow \mathbb{W}_{s}, \quad \mathcal{P}(\cdot)=\left(\cdot, \Phi_{s}\right), \quad \Phi_{s}=\left[\phi_{1} \phi_{2} \cdots \phi_{m}\right]^{T}, \\
& \mathcal{Q}: \mathbb{W} \rightarrow \mathbb{W}_{f}, \quad \mathcal{Q}(\cdot)=\left(\cdot, \Phi_{f}\right), \quad \Phi_{f}=\left[\phi_{m+1} \phi_{m+2} \cdots\right]^{T},
\end{aligned}
$$

to project the infinite-dimensional system representation of (2) to partitioned sets of ODEs of the vectorized slow and fast eigenmodes,

$$
\begin{aligned}
& \dot{x}_{s}(t)=A_{s} x_{s}(t)+f_{s}\left(x_{s}(t), x_{f}(t)\right)+b_{s} u(t), \quad x_{s}(0)=\mathcal{P} x_{0}, \\
& \dot{x}_{f}(t)=A_{f} x_{f}(t)+f_{f}\left(x_{s}(t), x_{f}(t)\right)+b_{f} u(t), \quad x_{f}(0)=Q x_{0},
\end{aligned}
$$

where $x(t)=x_{s}(t) \oplus x_{f}(t), A_{s}=\mathcal{P} A=\operatorname{diag}\left\{\lambda_{i}\right\}_{i=1}^{m}, A_{f}=Q A=\operatorname{diag}\left\{\lambda_{i}\right\}_{i=m+1}^{\infty}, f_{s}=\mathcal{P} f, f_{f}=Q f$, $b_{s}=\mathcal{P} b, b_{f}=Q b$ and $\operatorname{diag}\{\cdot\}$ denotes the diagonal matrix with diagonal elements. Then the partitioned 
infinite dimensional ODEs of (6) can be approximated by

$$
\dot{x}_{s}(t)=A_{s} x_{s}(t)+f_{s}\left(x_{s}(t), 0\right)+b_{s} u(t), \quad x_{s}(0)=\mathcal{P} x_{0}
$$

after a short period of time, $t_{b}$, when $x_{f} \rightarrow 0$, by applying singular perturbation analysis $[5,7,9,21]$ and considering Tykhonov's theorem for solution convergence of systems that consist of slow and fast subsystems [46]. The time-scale separation between slow and fast dynamics of the DPS modeled by PDEs ensures that a controller which exponentially stabilizes the closed-loop finite dimensional approximation also exponentially stabilizes the closed-loop infinite-dimensional system [21].

Remark 2. We may replace the approximate observability and controllability assumption of the infinitedimensional system of (2) formally addressed in Assumption 1, by observability and controllability of the system approximation (slow subsystem) of (7) [25] when the fast subsystem in (6) is exponentially stable and the control input is bounded and changes at a rate that is in the slow time-scale.

Remark 3. A lower bound for the relaxation time, $t_{b}$, required by the fast dynamics of the system to relax, can be identified by singular perturbation analysis [5, 9, 21].

\section{Spatiotemporal dynamic shaping using sliding mode controller designs}

\subsection{Dynamic shaping error formulation}

To address the spatiotemporal dynamic shaping problem of the DPSs described by PDE system of (1), we consider a desired spatiotemporal dynamics described by a target PDE with the same spatial differential operator,

$$
\begin{aligned}
& \frac{\partial}{\partial t} X_{d}(z, t)=\mathcal{A}_{n}(z) X_{d}(z, t)+F^{\prime}\left(z, t, X_{d}(z, t)\right), \\
& q\left(X_{d}, \frac{\partial X_{d}}{\partial z}, \ldots, \frac{\partial^{n-1} X_{d}}{\partial z^{n-1}}\right)=0 \text { on } \partial \Omega, \\
& X_{d}(z, 0)=X_{d 0}(z) .
\end{aligned}
$$

According to same spatial differential operator and boundary conditions, both the system and target PDEs have the same set of dominant eigenfunctions which can be applied to approximate the spatiotemporal states as follows,

$$
\begin{aligned}
& X(z, t) \approx x_{s}^{T}(t) \Phi_{s}(z), \\
& X_{d}(z, t) \approx x_{d s}^{T}(t) \Phi_{s}(z) .
\end{aligned}
$$


Note that such approximations are quite accurate after a short relaxation time when the fast dynamics stabilize. Then the spatiotemporal dynamic shaping error with respect to slow system dynamics can be formulated by

$$
E(z, t)=X(z, t)-X_{d}(z, t) \approx\left(x_{s}^{T}(t)-x_{d s}^{T}(t)\right) \Phi_{s}(z)=e^{T}(t) \Phi_{s}(z)
$$

where $e(t)=x_{s}(t)-x_{d s}(t)$ indicates the vector of modal errors.

By applying MOR via Galerkin projection as it was discussed in Section 3, we can discretize the target PDE to the following set of ODEs,

$$
\dot{x}_{d s}(t)=A_{s} x_{d s}(t)+f_{s}^{\prime}\left(t, x_{d s}(t)\right), x_{d s}(0)=\mathcal{P} x_{d 0},
$$

which describes the slow modal dynamics of the desired spatiotemporal behavior. The modal tracking error dynamics can then be derived by subtracting the ODEs of (7) and (11),

$$
\dot{e}(t)=A_{s} e(t)+f_{s}\left(e(t), x_{d s}(t)\right)-f_{s}^{\prime}\left(t, x_{d s}(t)\right)+b_{s} u(t), \quad e(0)=\mathcal{P} e_{0}
$$

where $e_{0}=x_{0}-x_{d 0}$. Thus the spatiotemporal dynamic shaping problem of the transport-reaction processes described by (1) can be addressed via error dynamics regulation of the low-dimensional ODEs of (12).

Remark 4. Note that the fast dynamics must be exponentially stable for the specific method to be applicable even for unstable target PDEs.

Remark 5. We may directly apply the proposed MOR to the PDE of the spatiotemporal dynamic shaping offset which is formulated by subtracting the governing PDE of (1) from the target of (8). Such alternative approach results to the same modal tracking error dynamics as (12).

Remark 6. In theory, we can reshape the system to any desired spatiotemporal response, in essence by canceling out the original dynamics of the system and replacing them with the intended dynamics of target. There are some considerations with allowable original and target dynamics. The target spatiotemporal dynamics must be bounded to avoid possible issues that arise in designing controller structures with unbounded actions. Since in the proposed control approach the desired spatiotemporal behavior described by the target PDE must have the same spatial differential operator and boundary conditions as the process PDE, the nonlinear functions of $F$ and $F^{\prime}$ are the only sources of differences in the governing and target dynamics. The most important restriction of the proposed approach is choosing a bounded target nonlinear functions $F^{\prime}$ and a stable target PDE. In addition, the locations of the actuators must satisfy the controllability requirements of error dynamics. Even when bounded, $F^{\prime}$ should not be introducing an 
oscillatory behavior in the target system with frequency that falls in the separation gap of the slow and fast dynamics; otherwise it will introduce a model reduction error that can also affect the tracking of the desired spatiotemporal dynamics.

\subsection{Sliding mode controller design}

Consider the nonlinear dynamical modal error system of (12),

$$
\dot{e}=A_{s} e+f_{s}\left(e, x_{d s}\right)-f_{s}^{\prime}\left(t, x_{d s}\right)+b_{s} u
$$

where $e=\left[e_{1}, e_{2}, \ldots, e_{m}\right]^{T} \in \mathbb{R}^{m}$ and $u=\left[u_{1}, u_{2}, \ldots, u_{l}\right]^{T} \in \mathbb{R}^{l}$. The objective is designing an outputfeedback control law, $u(t)$, which regulates the error dynamics at the origin. By stabilizing the error, such regulator enforces the system dominant eigenmodes to follow the target eigenmodes. According to the dynamical modal error system dimension, we require at least $m$ manipulated inputs (i.e. $l \geq m$ ) to stabilize the tracking error and force the system spatiotemporal dynamics to follow target dynamics with a general nonlinear term $[31,34]$.

To reach such objective a sliding mode control approach is applied. The controller synthesis includes two steps: (1) Choosing a manifold (reduced-order subspace), also known as sliding surface, which describes the desired dynamic behavior. (2) Designing the feedback control law which forces the tracking error trajectory to confine to the sliding surface and slides along it [27,63]. Thus the problem of dynamic modal response shaping $a \rightarrow \tilde{a}$ is equivalent to "approaching to the sliding surface and remaining on it". The time-varying sliding surface, $S(t)$, is defined by the scalar equation of $s(e)=0$ which must guarantee the system control objective in regulating the tracking error dynamics,

$$
S=\left\{e \in \mathbb{R}^{m}: s(e)=0\right\}
$$

The switching function of $s(e)$ is a distance measurement which indicates how far the system eigenmodes are from the target eigenmodes. It can be simply defined as the following proportional-integral form,

$$
s(e)=e+\Pi \int e d t
$$


where $\Pi=\operatorname{diag}\left\{\pi_{i}\right\}_{i=1}^{m}$. To assure the stability of sliding mode dynamic, the diagonal components of $\Pi$ must have negative real parts, i.e, $\operatorname{Re}\left(\pi_{i}\right)<0$ for $i=1, \ldots, m$. The places of such components in the left half-plane determine the performance of the sliding mode controller in stabilizing the tracking error dynamics.

Due to the discontinuous nature of the resulting sliding mode control action, the existence and uniqueness of the closed-loop system solution can not be verified by the Picard-Lindelöf theorem [60]. Such switching discontinuous closed-loop dynamics must be analyzed using Filippov theorem [29, 67] which states the resulting closed-loop system that slides along $s(e)=0$, can be approximated by a smooth dynamics which is described using

$$
\dot{s}(e)=0
$$

under a continuous control design $[41,56]$. To formulate the sliding mode controller design we consider the Lyapunov function in the quadratic form of

$$
V(s)=\frac{1}{2} s^{T} s
$$

By considering the above Lyapunov function, the asymptotic stability can be obtained by

$$
\dot{V}(s)=s^{T} \dot{s} \leq 0
$$

Then using Filippov's construction of the equivalent dynamics, the control action can be computed by considering the smooth dynamics of $\dot{s}=0$, which

$$
\dot{s}=\dot{e}+\Pi e=A_{s} e+f_{s}\left(e, x_{d s}\right)-f_{s}^{\prime}\left(t, x_{d s}\right)+b_{s} u_{e q}+\Pi e=0
$$

As a result the equivalent control law takes the following form

$$
u_{e q}=-b_{s}^{\perp}\left(\left(A_{s}+\Pi\right) e+f_{s}\left(e, x_{d s}\right)-f_{s}^{\prime}\left(t, x_{d s}\right)\right)
$$


where $b_{s}^{\perp}=b_{s}^{T}\left(b_{s} b_{s}^{T}\right)^{-1}$ identifies the Moore-Penrose pseudo-inverse [52]. Note that $b_{s}^{\perp}=b_{s}^{-1}$ for $m=l$. In order to satisfy the sliding condition we consider the control law as

$$
u=u_{e q}-b_{s}^{\perp} \eta \operatorname{sign}(s)
$$

where $\eta>0$ and $\operatorname{sign}(\cdot)$ denotes the sign function. Then we obtain

$$
\dot{V}(s)=s^{T} \dot{s}=-\eta s^{T} \operatorname{sign}(s),
$$

where $\dot{V}(s)<0$ and $\dot{V}(0)=0$, thus the closed-loop system is locally asymptotically stable in the Lyapunov sense $[41,56]$.

Remark 7. The control law of (19) was derived by Lyapunov direct method which is a typical controller design approach for general nonlinear systems [41, 56]. First we introduced a Lyapunov function candidate in the form of (15). Using Fillippov's construction of the equivalent dynamics we found an equivalent controller formula for which the time derivative of the Lyapunov function candidate is equal to zero. Then we obtained the control law by subtracting a positive term which contains a tuning parameter from the equivalent control formula to obtain the negative sign for Lyapunov candidate time derivative which guarantees the closed-loop process stability.

Remark 8. The positive controller tuning parameter of $\eta$ allows a certain degree of flexibility in spatiotemporal dynamic shaping of the studied transport-reaction process. Large values of $\eta$ force the Lyapunov function time derivative, $\dot{V}$, to be more negative and therefore generate a faster transient response at the cost of larger control action.

Remark 9. In practice, implementation of the sliding mode controller with the discontinuous nonlinearity in the form of sign(.), presents the chattering phenomenon ("zig-zag" motion) due to fast switching fluctuations across the sliding surface. Chattering which involves high control activity is undesirable in practice. Such high activities may excite high frequency dynamics neglected in the process modeling step $[41,56]$.

\subsection{Dynamic observer design}

For the desired spatiotemporal dynamics which is described by (8) and approximated by the slow dynamics of (11) we have access to the dominant eigenmodes, $x_{d s}$. However, to compute the tracking error vectors, $e$, and implement the control law of (19), we need to estimate the values of the system dominant 
eigenmodes of $x_{s}$. For such estimation purpose, a Luenberger-type dynamic observer is synthesized based on the system ROM,

$$
\begin{aligned}
& \dot{\hat{x}}_{s}=A_{s} \hat{x}_{s}+f_{s}\left(\hat{x}_{s}\right)+b_{s} u+\Theta\left(y-C \hat{x}_{s}\right), \\
& y=C x_{s},
\end{aligned}
$$

where

$$
C=\left[\begin{array}{cccc}
\phi_{1}\left(\omega_{1}\right) & \phi_{2}\left(\omega_{1}\right) & \cdots & \phi_{m}\left(\omega_{1}\right) \\
\phi_{1}\left(\omega_{2}\right) & \phi_{2}\left(\omega_{2}\right) & \cdots & \phi_{m}\left(\omega_{2}\right) \\
\vdots & \vdots & \ddots & \vdots \\
\phi_{1}\left(\omega_{p}\right) & \phi_{2}\left(\omega_{p}\right) & \cdots & \phi_{m}\left(\omega_{p}\right)
\end{array}\right]
$$

and $\hat{x}_{S}$ denotes the estimated dominant eigenmodes of the system, $\Theta$ presents the dynamic observer gain matrix and $\omega=\left[\begin{array}{llll}\omega_{1} & \omega_{2} & \cdots & \omega_{p}\end{array}\right]^{T}$ is the vector of locations of continuous measurements, $y$. The modal observation error dynamics can then be formulated as follows

$$
\dot{e}_{o}=\left(A_{s}-\Theta C\right) e_{o}+f_{s}\left(e_{o}+\hat{x}_{s}\right)-f_{s}\left(\hat{x}_{s}\right)
$$

where the vector of observation error is defined by $e_{o}=x_{s}-\hat{x}_{s}$. Assuming the principle of separation between control and observation holds $[3,19]$, we consider the observation Lyapunov function (OLF) in the standard quadratic form

$$
V_{o}=\frac{1}{2} e_{o}^{T} P e_{o}
$$

where $P$ is a symmetric positive definite matrix with a bounded norm, $\|P\| \leq K_{1}$. The time derivative of the OLF is obtained as follows

$$
\begin{aligned}
& \dot{V}_{o}=\frac{1}{2}\left(\dot{e}_{o}^{T} P e_{o}+e_{o}^{T} P \dot{e}_{o}\right) \\
& =\frac{1}{2}\left(\left[\left(A_{s}-\Theta C\right) e_{o}+f_{s}\left(e_{o}+\hat{x}_{s}\right)-f_{s}\left(\hat{x}_{s}\right)\right]^{T} P e_{o}+e_{o}^{T} P\left[\left(A_{s}-\Theta C\right) e_{o}+f_{s}\left(e_{o}+\hat{x}_{s}\right)-f_{s}\left(\hat{x}_{s}\right)\right]\right) \\
& =\frac{1}{2}\left(\left[e_{o}^{T}\left(A_{s}-\Theta C\right)^{T}+\left(f_{s}\left(e_{o}+\hat{x}_{s}\right)-f_{s}\left(\hat{x}_{s}\right)\right)^{T}\right] P e_{o}+e_{o}^{T} P\left[\left(A_{s}-\Theta C\right) e_{o}+f_{s}\left(e_{o}+\hat{x}_{s}\right)-f_{s}\left(\hat{x}_{s}\right)\right]\right) \\
& =\frac{1}{2}\left(e_{o}^{T}\left[\left(A_{s}-\Theta C\right)^{T} P+P\left(A_{s}-\Theta C\right)\right] e_{o}+e_{o}^{T} P\left[f_{s}\left(e_{o}+\hat{x}_{s}\right)-f_{S}\left(\hat{x}_{s}\right)\right]+\left(e_{o}^{T} P\left[f_{s}\left(e_{o}+\hat{x}_{s}\right)-f_{s}\left(\hat{x}_{s}\right)\right]\right)^{T}\right) \text {. }
\end{aligned}
$$


Then the dynamic observer gain matrix, $\Theta$, must be identified subject to $\dot{V}_{o}<0$ which indicates the observation stability. Due to the Lipschitz property of the nonlinear function of $F$ in the DPS of (1) and bounded nature of the eigenfunctions and their adjoints, the nonlinear function of $f_{s}$ in the system approximation of (7) is also Lipschitz continuous,

$$
\left\|f_{s}\left(e_{o}+\hat{x}_{s}\right)-f_{s}\left(\hat{x}_{s}\right)\right\| \leq K_{2}\left\|e_{o}\right\|
$$

where $K_{2}$ denotes the Lipschitz upper bound gain. From (24) and (25) and using Cauchy-Schwarz inequality we obtain that

$$
\begin{aligned}
e_{o}^{T} P\left[f_{s}\left(e_{o}+\hat{x}_{s}\right)-f_{s}\left(\hat{x}_{s}\right)\right]+\left(e_{o}^{T} P\left[f_{s}\left(e_{o}+\hat{x}_{s}\right)-f_{s}\left(\hat{x}_{s}\right)\right]\right)^{T} & \leq 2\left\|e_{o}^{T} P\left[f_{s}\left(e_{o}+\hat{x}_{s}\right)-f_{s}\left(\hat{x}_{s}\right)\right]\right\| \\
& \leq 2\left\|e_{o}^{T}\right\|\|P\|\left\|f_{s}\left(e_{o}+\hat{x}_{s}\right)-f_{s}\left(\hat{x}_{s}\right)\right\| \\
& \leq 2 K_{1} K_{2}\left\|e_{o}^{T}\right\|\left\|e_{o}\right\| \\
& =K\left\|e_{o}\right\|^{2}=K e_{o}^{T} e_{o},
\end{aligned}
$$

where $K=K_{1} K_{2}$.

By applying the inequality of (26), we conclude that if

$$
e_{o}^{T}\left[\left(A_{s}-\Theta C\right)^{T} P+P\left(A_{s}-\Theta C\right)\right] e_{o}+K e_{o}^{T} e_{o}<0 .
$$

then $\dot{V}_{o}<0$ and $\dot{V}(0)=0$. Thus

$$
e_{o}^{T}\left[\left(A_{s}-\Theta C\right)^{T} P+P\left(A_{s}-\Theta C\right)+K I\right] e_{o}<0
$$

where $\left(A_{s}-\Theta C\right)^{T} P+P\left(A_{s}-\Theta C\right)+K I<0$ guarantees the asymptotic stability of the observation error dynamics. We can then address the dynamic observer synthesis problem via a standard linear matrix inequality (LMI) problem

$$
\left(A_{s}-\Theta C\right)^{T} P+P\left(A_{s}-\Theta C\right)+K I<-P Y P-P \Theta Z \Theta^{T} P
$$


where $Y$ and $Z$ are the symmetric positive definite weighting matrices. From the inequality of (29) we obtain

$$
\begin{aligned}
& \left(A_{s}^{T}-C^{T} \Theta^{T}\right) P+P\left(A_{s}-\Theta C\right)+K I+P Y P+P \Theta Z \Theta^{T} P<0 \\
& \Rightarrow A_{s}^{T} P+P A_{s}-(P \Theta C)^{T}-P \Theta C+P Y P+P \Theta Z \Theta^{T} P+K I<0 \\
& \Rightarrow A_{s}^{T} P+P A_{s}-(U C)^{T}-U C+P Y P+U Z U^{T}+K I<0 \\
& \Rightarrow A_{s}^{T} P+P A_{s}+\left(U^{T}-Z^{-1} C\right)^{T} Z\left(U^{T}-Z^{-1} C\right)-C^{T} Z^{-1} C+P Y P+K I<0,
\end{aligned}
$$

where $U=P \Theta$. Then we reduce the degrees of freedom in the inequality by setting $U=C^{T} Z^{-T}$,

$$
A_{s}^{T} P+P A_{s}-C^{T} Z^{-1} C+P Y P+K I<0 .
$$

Using the Schur Complement Lemma we represent the inequality of (31) in the following standard form [18],

$$
\left[\begin{array}{cc}
P A_{s}+A_{s}^{T} P-C^{T} Z^{-1} C+K I & P \\
P & -Y^{-1}
\end{array}\right]<0 .
$$

The observer gain matrix can be computed by minimizing the trace of $P^{-1}$ subject to the LMI constraint of (32),

$$
\Theta=P^{-1} C^{T} Z^{-1}
$$

A detailed discussion on the LMI-constrained optimization problem can be found in [54].

\section{Application to thermal dynamic shaping in a tubular reactor}

In this section, we illustrate the effectiveness of the proposed output feedback sliding mode control on spatiotemporal dynamic shaping of a typical transport-reaction process example. In the first part we present the mathematical model of the thermal dynamics in a tubular chemical reactor. The desired spatiotemporal behavior is described in the second part. Then the tailored MOR and control structure are presented for the specific dynamic shaping problem in the third part of this section. Finally, the closedloop simulation results are provided to assess the system performance under the proposed output feedback sliding mode control structure. 


\subsection{System description}

We consider a tubular chemical flow reactor [21] where an irreversible exothermic reaction of the zero-th order takes place. As presented in Figure 1, a limited set of $l$ cooling jackets are employed as the manipulated inputs to remove the heat from the reactor and manage the thermal energy along the reactor length as time evolves. The spatiotemporal thermal dynamics in the presence of temperature-dependent reaction rate is derived from the energy balance which takes the form of the following dissipative PDE,

$$
\begin{array}{ll}
\frac{\partial T}{\partial t}=\frac{k}{\rho C_{p}} \frac{\partial^{2} T}{\partial z^{2}}-v \frac{\partial T}{\partial z}+\frac{(-\Delta H)}{\rho C_{p}} r_{o} \exp \left(\frac{-E}{R T}\right)-\sum_{j=1}^{l} \mathcal{B}_{j}(z) \frac{h A_{c}}{\rho C_{p}}\left(T-T_{c, j}\right), \\
z=0: \quad \frac{\partial T}{\partial z}=\frac{\rho C_{p} v}{k}\left(T-T_{f}\right), \\
z=L: \quad \frac{\partial T}{\partial z}=0, \\
t=0: \quad T=T_{0},
\end{array}
$$

where

$$
\begin{aligned}
& T: \text { temperature of the fluid inside the reactor, } \\
& t: \text { time, } \\
& z \in[0, L]: \text { spatial coordinate, } \\
& L: \text { reactor length, } \\
& k: \text { thermal conductivity, } \\
& \rho: \text { density, } \\
& C_{p}: \text { heat capacity, } \\
& v: \text { axial velocity, } \\
& (-\Delta H): \text { heat of reaction, } \\
& r_{0}: \text { pre-exponential reaction constant, } \\
& E: \text { activation energy, } \\
& h: \text { heat transfer coefficient between reactor and cooling jacket, } \\
& A_{c}: \text { cooling surface area, } \\
& T_{c, j}: \text { cooling jackets temperatures, } \\
& T_{f}: \text { feed temperature, }
\end{aligned}
$$




\section{$l$ : number of cooling jackets, \\ $\mathcal{B}_{j}(z)$ : spatial distribution of the $j^{\text {th }}$ cooling jacket, \\ $T_{0}$ : initial temperature profile.}

We reformulate the PDE set of (34) in dimensionless form and homogenize the left boundary condition to employ the proposed MOR. To homogenize the boundary condition we induce the non-homogeneous part in the PDE by standard Dirac function. The resulting dimensionless PDE takes the following form,

$$
\begin{aligned}
& \frac{\partial \bar{T}}{\partial \bar{t}}=\frac{1}{P e} \frac{\partial^{2} \bar{T}}{\partial \bar{z}^{2}}-\frac{\partial \bar{T}}{\partial \bar{z}}+B_{t} \exp \left(\frac{\gamma \bar{T}}{1+\bar{T}}\right)+B_{c} \sum_{j=1}^{l} \mathcal{B}_{j}(\bar{z})\left(\bar{u}_{i}-\bar{T}\right)+\delta(\bar{z}-0) \bar{T}_{f} \\
& \bar{z}=0: \quad \frac{\partial \bar{T}}{\partial \bar{z}}=P e \bar{T}, \\
& \bar{z}=1: \quad \frac{\partial \bar{T}}{\partial \bar{z}}=0, \\
& \bar{t}=0: \quad \bar{T}=0,
\end{aligned}
$$

where

$$
\begin{array}{ll}
\bar{t}=\frac{t v}{L}, \quad \bar{z}=\frac{z}{L}, \quad \bar{T}=\frac{T-T_{0}}{T_{0}}, & P e=\frac{\rho C_{p} v L}{k}, \\
\gamma=\frac{E}{R T_{0}}, \quad \bar{u}_{j}=\frac{T_{c, j}-T_{0}}{T_{0}}, & \bar{T}_{f}=\frac{T_{f}-T_{0}}{T_{0}}, \\
B_{t}=\frac{(-\Delta H) r_{o} \exp \left(-\frac{E}{R T_{0}}\right) L}{\rho C_{p} T_{0} v}, & B_{c}=\frac{h A_{c} L}{\rho C_{p} v} .
\end{array}
$$

A typical diffusion-convection-reaction process with the same spatial differential operator is considered as the target PDE which describes the desired spatiotemporal dynamics,

$$
\begin{array}{ll}
\frac{\partial \bar{T}_{d}}{\partial \bar{t}}=\frac{1}{P e} \frac{\partial^{2} \bar{T}_{d}}{\partial \bar{z}^{2}}-\frac{\partial \bar{T}_{d}}{\partial \bar{z}}+\alpha_{3} \bar{T}_{d}^{3}+\alpha_{2} \bar{T}_{d}^{2}+\alpha_{1} \bar{T}_{d}+\alpha_{0}+\beta_{2} \cos (0.5 \pi \bar{t})+\beta_{1} \sin (0.5 \pi \bar{t}), & \frac{\partial \bar{T}_{d}}{\partial \bar{z}}=P e \bar{T}_{d}, \\
\bar{z}=0: & \frac{\partial \bar{T}_{d}}{\partial \bar{z}}=0, \\
\bar{z}=1: & \bar{T}_{d}=0 .
\end{array}
$$

Remark 10. The time-varying target PDE of (36) which is set by choosing the bounded time-varying nonlinear term, presents a permanent oscillatory behavior in spatiotemporal thermal dynamics of the tubular reactor. 


\subsection{Model order reduction and output feedback control structure}

For MOR of the system and target PDEs we require the eigenfunctions which must be computed by solving the following eigenproblem of the system and target spatial differential operator,

$$
\begin{aligned}
& \frac{1}{P e} \frac{d^{2} \phi_{i}}{d \bar{z}^{2}}-\frac{d \phi_{i}}{d \bar{z}}=\lambda_{i} \phi_{i}, \\
& \bar{z}=0: \frac{d \phi_{i}}{d \bar{z}}=P e \phi_{i} \\
& \bar{z}=1: \frac{d \phi_{i}}{d \bar{z}}=0 .
\end{aligned}
$$

where $i=1,2, \ldots, \infty$. The solution of above eigenvalue-eigenfunction problem takes the following form $[21,43,45]$,

$$
\begin{aligned}
& \lambda_{i}=-\left(\frac{\alpha_{i}^{2}}{P e}+\frac{P e}{4}\right), \quad \tan \left(\alpha_{i}\right)=\frac{P e \alpha_{i}}{\alpha_{i}^{2}-\left(\frac{P e}{2}\right)^{2}}, \\
& \phi_{i}(\bar{z})=\xi_{i} \exp \left(\frac{P e \bar{z}}{2}\right)\left[\cos \left(\alpha_{i} \bar{z}\right)+\frac{P e}{2 \alpha_{i}} \sin \left(\alpha_{i} \bar{z}\right)\right], \\
& \xi_{i}=\left(\int_{0}^{1}\left[\cos \left(\alpha_{i} \bar{z}\right)+\frac{P e}{2 \alpha_{i} \bar{z}} \sin \left(\alpha_{i} \bar{z}\right)\right]^{2} d \bar{z}\right)^{-\frac{1}{2}} .
\end{aligned}
$$

The resulting eigenfunctions are not self-adjoint because the spatial differential operator of $\frac{1}{P e} \frac{\partial^{2}}{\partial \bar{z}^{2}}-\frac{\partial}{\partial \bar{z}}$ is non-self-adjoint; then to apply the Galerkin projection we must define the adjoint eigenfunctions

$$
\phi_{i}^{*}(\bar{z})=\exp (-P e \bar{z}) \phi_{i}(\bar{z})
$$

which satisfy the orthonormal property of (4). By considering the set of $m$ dominant eigenfunctions of $\Phi_{s}=\left[\begin{array}{llll}\phi_{1} & \phi_{2} & \cdots & \phi_{m}\end{array}\right]^{T}$, we approximate the system and desired dimensionless temperatures

$$
\begin{aligned}
& \bar{T}(\bar{z}, \bar{t}) \approx \sum_{i=1}^{m} a_{i}(\bar{t}) \phi_{i}(\bar{z}) \\
& \bar{T}_{d}(\bar{z}, \bar{t}) \approx \sum_{i=1}^{m} \tilde{a}_{i}(\bar{t}) \phi_{i}(\bar{z})
\end{aligned}
$$


where $\sigma=\frac{\left|\lambda_{1}\right|}{\left|\lambda_{m+1}\right|}$ has a small positive value. Then by employing Galerkin's method, the ROMs of the system and target PDEs take the following modal forms,

$$
\begin{aligned}
& \dot{a}_{k}=\lambda_{k} a_{k}+B_{t} \int_{0}^{1} \phi_{k}^{*} \exp \left(\frac{\gamma \sum_{i=1}^{m} a_{i} \phi_{i}}{1+\sum_{i=1}^{m} a_{i} \phi_{i}}\right) d \bar{z}+\phi_{k}^{*}(0) \bar{T}_{f}-B_{c} \sum_{j=1}^{l}\left(\int_{0}^{1} \mathcal{B}_{j} \phi_{k}^{*} \sum_{i=1}^{m} a_{i} \phi_{i} d \bar{z}\right)+B_{c} \sum_{j=1}^{l}\left(\int_{0}^{1} \mathcal{B}_{j} \phi_{k}^{*} d \bar{z}\right) \bar{u}_{j}, \\
& \dot{\tilde{a}}_{k}=\lambda_{k} \tilde{a}_{k}+\int_{0}^{1}\left(\alpha_{3}\left(\sum_{i=1}^{m} \tilde{a}_{i} \phi_{i}\right)^{3}+\alpha_{2}\left(\sum_{i=1}^{m} \tilde{a}_{i} \phi_{i}\right)^{2}+\alpha_{0}+\beta_{2} \cos (0.5 \pi \bar{t})+\beta_{1} \sin (0.5 \pi \bar{t})\right) \phi_{k}^{*} d \bar{z}+\alpha_{1} \tilde{a}_{k}
\end{aligned}
$$

for $k=1,2, \ldots, m$. Then the above ROMs can be summarized in the following abstract forms

$$
\begin{aligned}
& \dot{a}=A a+f(a)+B u, \\
& \dot{\tilde{a}}=A \tilde{a}+f^{\prime}(\bar{t}, \tilde{a}),
\end{aligned}
$$

where

$$
\begin{aligned}
& a=\left[\begin{array}{c}
a_{1} \\
a_{2} \\
\vdots \\
a_{m}
\end{array}\right], \quad \tilde{a}=\left[\begin{array}{c}
\tilde{a}_{1} \\
\tilde{a}_{2} \\
\vdots \\
\tilde{a}_{m}
\end{array}\right], \quad A=\left[\begin{array}{cccc}
\lambda_{1} & 0 & \cdots & 0 \\
0 & \lambda_{2} & \cdots & 0 \\
\vdots & \vdots & \ddots & \vdots \\
0 & 0 & \cdots & \lambda_{m}
\end{array}\right], \quad u=\left[\begin{array}{c}
\bar{u}_{1} \\
\bar{u}_{2} \\
\vdots \\
\bar{u}_{l}
\end{array}\right], \\
& f(a)=\left[\begin{array}{c}
B_{t} \int_{0}^{1} \phi_{1}^{*} \exp \left(\frac{\gamma \sum_{i=1}^{m} a_{i} \phi_{i}}{1+\sum_{i=1}^{m} a_{i} \phi_{i}}\right) d \bar{z}+\phi_{1}^{*}(0) \bar{T}_{f}-B_{c} \sum_{j=1}^{l}\left(\int_{0}^{1} \mathcal{B}_{j} \phi_{1}^{*} \sum_{i=1}^{m} a_{i} \phi_{i} d \bar{z}\right) \\
B_{t} \int_{0}^{1} \phi_{2}^{*} \exp \left(\frac{\gamma \sum_{i=1}^{m} a_{i} \phi_{i}}{1+\sum_{i=1}^{m} a_{i} \phi_{i}}\right) d \bar{z}+\phi_{2}^{*}(0) \bar{T}_{f}-B_{c} \sum_{j=1}^{l}\left(\int_{0}^{1} \mathcal{B}_{j} \phi_{2}^{*} \sum_{i=1}^{m} a_{i} \phi_{i} d \bar{z}\right) \\
\vdots \\
B_{t} \int_{0}^{1} \phi_{m}^{*} \exp \left(\frac{\gamma \sum_{i=1}^{m} a_{i} \phi_{i}}{1+\sum_{i=1}^{m} a_{i} \phi_{i}}\right) d \bar{z}+\phi_{m}^{*}(0) \bar{T}_{f}-B_{c} \sum_{j=1}^{l}\left(\int_{0}^{1} \mathcal{B}_{j} \phi_{m}^{*} \sum_{i=1}^{m} a_{i} \phi_{i} d \bar{z}\right)
\end{array}\right],
\end{aligned}
$$




$$
\begin{gathered}
B=\left[\begin{array}{cccc}
B_{c} \int_{0}^{1} \mathcal{B}_{1} \phi_{1}^{*} d \bar{z} & B_{c} \int_{0}^{1} \mathcal{B}_{2} \phi_{1}^{*} d \bar{z} & \cdots & B_{c} \int_{0}^{1} \mathcal{B}_{l} \phi_{1}^{*} d \bar{z} \\
B_{c} \int_{0}^{1} \mathcal{B}_{1} \phi_{2}^{*} d \bar{z} & B_{c} \int_{0}^{1} \mathcal{B}_{2} \phi_{2}^{*} d \bar{z} & \cdots & B_{c} \int_{0}^{1} \mathcal{B}_{l} \phi_{2}^{*} d \bar{z} \\
\vdots & \vdots & \ddots & \vdots \\
B_{c} \int_{0}^{1} \mathcal{B}_{1} \phi_{m}^{*} d \bar{z} & B_{c} \int_{0}^{1} \mathcal{B}_{2} \phi_{m}^{*} d \bar{z} & \cdots & B_{c} \int_{0}^{1} \mathcal{B}_{l} \phi_{m}^{*} d \bar{z}
\end{array}\right], \\
f^{\prime}(\bar{t}, \tilde{a})=\left[\begin{array}{c}
\int_{0}^{1}\left(\alpha_{3}\left(\sum_{i=1}^{m} \tilde{a}_{i} \phi_{i}\right)^{3}+\alpha_{2}\left(\sum_{i=1}^{m} \tilde{a}_{i} \phi_{i}\right)^{2}+\alpha_{0}+\beta_{2} \cos (0.5 \pi \bar{t})+\beta_{1} \sin (0.5 \pi \bar{t})\right) \phi_{1}^{*} d \bar{z}+\alpha_{1} \tilde{a}_{1} \\
\int_{0}^{1}\left(\alpha_{3}\left(\sum_{i=1}^{m} \tilde{a}_{i} \phi_{i}\right)^{3}+\alpha_{2}\left(\sum_{i=1}^{m} \tilde{a}_{i} \phi_{i}\right)^{2}+\alpha_{0}+\beta_{2} \cos (0.5 \pi \bar{t})+\beta_{1} \sin (0.5 \pi \bar{t})\right) \phi_{2}^{*} d \bar{z}+\alpha_{1} \tilde{a}_{2} \\
\int_{0}^{1}\left(\alpha_{3}\left(\sum_{i=1}^{m} \tilde{a}_{i} \phi_{i}\right)^{3}+\alpha_{2}\left(\sum_{i=1}^{m} \tilde{a}_{i} \phi_{i}\right)^{2}+\alpha_{0}+\beta_{2} \cos (0.5 \pi \bar{t})+\beta_{1} \sin (0.5 \pi \bar{t})\right) \phi_{m}^{*} d \bar{z}+\alpha_{1} \tilde{a}_{m}
\end{array}\right] .
\end{gathered}
$$

By considering the modal error as $e=a-\tilde{a}$, the error dynamics can be formulated by

$$
\dot{e}=A e+f(e, \tilde{a})-f^{\prime}(\bar{t}, \tilde{a})+B u
$$

Then the sliding mode controller and dynamics observer structures take the following form

$$
\begin{aligned}
& u=-B^{\perp}\left((A+\Pi) \hat{e}+f(\hat{e}, \tilde{a})-f^{\prime}(\bar{t}, \tilde{a})+\eta \operatorname{sign}\left(\hat{e}+\Pi \int_{0}^{t} \hat{e} d t\right)\right), \\
& \dot{\hat{a}}=A \hat{a}+f(\hat{a})+B u+\Theta(y-C \hat{a}), \\
& \hat{e}=\hat{a}-\tilde{a},
\end{aligned}
$$

where the dynamic observer gain matrix, $\Theta$, can be computed using the LMI-constrained optimization problem described in Section 4.3.

\subsection{Simulation results}

To simulate the system we set the following typical values for the process parameters: $P e=5, \gamma=3$, $B_{t}=0.4, B_{c}=0.5$ and $\bar{T}_{f}=0$. The dominant eigenvalues of the system are presented in Table 1 . We can 
easily recognize an order of magnitude separation between the 3 dominant eigenvalues and the remainder,

$\sigma=\frac{\operatorname{Re}\left(\left|\lambda_{1}\right|\right)}{\operatorname{Re}\left(\left|\lambda_{4}\right|\right)}=0.093$. The corresponding dominant eigenfunctions and their adjoints are presented in Figure 2 .

When considering the first three dominant eigenfunctions to discretize the system and desired PDEs, the resulting system and target approximations are of dimension $3(m=3)$. We set $l=3$ cooling jackets along the reactor length to shape the spatiotemporal temperature dynamics of the system. The spatial distributions of the control actuators are also described by $\mathcal{B}_{1}(\bar{z})=H(\bar{z}-0.1)-H(\bar{z}-0.2), \mathcal{B}_{2}(\bar{z})=$ $H(\bar{z}-0.4)-H(\bar{z}-0.5)$ and $\mathcal{B}_{3}(\bar{z})=H(\bar{z}-0.7)-H(\bar{z}-0.9)$, where $H(\cdot)$ indicates the standard Heaviside step function. We also consider two point sensors to measure the reactor temperature at $\omega=\left[\begin{array}{ll}0.3 & 0.6\end{array}\right]^{T}$ which can be employed by the dynamic observer to estimate the dominant eigenmodes of the system.

The entire system operation was partitioned into two time periods: (1) open-loop process operation, $\bar{t}_{o}=[0,8]$ when the controller was inactive, and (2) closed-loop process operation, $\bar{t}_{c}=(8,12]$ under the proposed controller design. The open-loop spatiotemporal profile of the dimensionless temperature and the temporal profile of its spatial $L_{2}$-norm while the controller was inactive $\left(\bar{u}_{i}=0\right.$ for $\left.i=1,2,3\right)$ are presented in Figure 3. It is observed that the dimensionless temperature converges to a nonuniform steady state profile. Figure 4 also shows the desired spatiotemporal profile and its $L_{2}$-norm where $\alpha_{0}=0.15$, $\alpha_{1}=0.05, \alpha_{2}=-0.1, \alpha_{3}=0.15, \beta_{1}=0.2$ and $\beta_{2}=-0.05$. We observe a permanent oscillatory behavior in the desired temperature spatiotemporal dynamics. The open-loop spatiotemporal profile of the shaping error and its spatial $L_{2}$-norm are presented in Figure 5.

The dynamic observer gain matrix was computed as follows,

$$
\Theta=\left[\begin{array}{cc}
0.20 & 0.64 \\
0.31 & -0.48 \\
-0.27 & -2.12
\end{array}\right]
$$

by solving the LMI-constrained optimization problem. Figure 6 presents the open-loop temporal profiles of the estimated dominant eigenmodes of the system, the desired dominant eigenmodes and the open-loop modal errors where the nondominant system and desired eigenmodes were negligible. 
To implement the proposed sliding mode controller we set $\eta=0.5$ for the controller parameter which guarantees the closed-loop system stability and performance. The spatiotemporal profile of the dimensionless reactor temperature and the temporal profile of its spatial $L_{2}$-norm is illustrated in Figure 7 for the entire process operation. The spatiotemporal profile of the shaping error and its $L_{2}$-norm are also presented in Figure 8. Note that the controller was only active during the closed-loop process operation of $\bar{t}_{c}=(8,12]$. We observe that the shaping error converges to zero and the system follows the desired spatiotemporal behavior under the proposed control approach.

The required control actions to stabilize the shaping error are shown in Figure 9. The zero control actions in the period of $\bar{t}_{o}=(0,8)$ illustrate the open-loop process operation. A sharp initial change in the control action is observed when the controller is activated at $\bar{t}=8$, but that is expected since this is a static controller design and there is significant offset from the desired behavior. We don't observe any significant chattering in the temporal profile of the control actions. An oscillatory behavior is observed in the controller signals due to the permanent oscillatory behavior of the target spatiotemporal dynamics. Finally, the temporal profile of the estimated dominant eigenmodes and the modal errors are presented in Figure 10 which illustrates the effectiveness of the sliding mode controller in regulating the shaping error and tracking the desired spatiotemporal dynamics.

\section{Conclusion}

The spatiotemporal dynamic shaping of semi-linear distributed parameter systems was investigated by regulating the error dynamics between the reduced order models (ROMs) of governing and target partial differential equations (PDEs). The required ROMs were derived by applying Galerkin projection to the describing PDEs. The spatiotemporal error dynamics between the governing and target ROMs were stabilized using a sliding mode controller design combined with a Luenberger-type dynamic observer to estimate the required states. The effectiveness of the proposed control structure was illustrated on thermal dynamic shaping of a tubular flow reactor. 


\section{Acknowledgment}

Financial support from the National Science Foundation, CMMI Award \# 13-00322 is gratefully acknowledged. 


\section{References}

[1] R.A. Adomaitis. A reduced-basis discretization method for chemical vapor deposition reactor simulation. Math Comput. Model, 38(1-2):159-175, 2003.

[2] A. Armaou and P.D. Christofides. Dynamic optimization of dissipative PDE systems using nonlinear order reduction. Chem. Eng. Sci., 57:5083-5114, 2002.

[3] A.N. Atassi and H.K. Khalil. A separation principle for the control of a class of nonlinear systems. IEEE Trans. Automatic Control, 46(5):742-746, 2001.

[4] D. Babaei Pourkargar and A. Armaou. Control of dissipative partial differential equation systems using APOD based dynamic observer designs. In Proceedings of the American Control Conference, 502-508, Washington, DC, 2013.

[5] D. Babaei Pourkargar and A. Armaou. Modification to adaptive model reduction for regulation of distributed parameter systems with fast transients. AIChE J., 59(12):4595-4611, 2013.

[6] D. Babaei Pourkargar and A. Armaou. Feedback control of linear distributed parameter systems via adaptive model reduction in the presence of device network communication constraints. In Proceedings of the American Control Conference, 1667-1673, Portland, OR, 2014.

[7] D. Babaei Pourkargar and A. Armaou. Geometric output tracking of nonlinear distributed parameter systems via adaptive model reduction. Chem. Eng. Sci., 116:418-427, 2014.

[8] D. Babaei Pourkargar and A. Armaou. Output tracking of spatiotemporal thermal dynamics in transport-reaction processes via adaptive model reduction. In Proceedings of the American Control Conference, 3364-3370, Portland, OR, 2014.

[9] D. Babaei Pourkargar and A. Armaou. APOD-based control of linear distributed parameter systems under sensor/controller communication bandwidth limitations. AIChE J., 61(2):434-447, 2015. 
[10] D. Babaei Pourkargar and A. Armaou. Control of spatially distributed processes with unknown transport-reaction parameters via two layer system adaptations. AIChE J., http://dx.doi.org/10.1002/aic.14852, 2015.

[11] D. Babaei Pourkargar and A. Armaou. Design of APOD-based switching dynamic observers and output feedback control for a class of nonlinear distributed parameter systems. Chem. Eng. Sci., http://dx.doi.org/10.1016/j.ces.2015.02.032, 2015.

[12] M.J. Balas. Nonlinear finite-dimensional control of a class of nonlinear distributed parameter systems using residual mode filters: A proof of local exponential stability. J. Math. Anal. Appl., 162:63-70, 1991.

[13] B. Bandyopadhyay and S. Janardhanan. Discrete-time sliding mode control: A Multirate output feedback approach, Lecture Notes in Control and Information Sciences. Springer-Verlag, Berlin, 2006.

[14] G. Bartolini, A. Ferrara, and S. Spurgeon (Eds.). Special issue: New trends in sliding mode control. Int. J. Robust Nonlinear Control, 7(4), 1997.

[15] A. Bartoszewicz, O. Kaynak, and V.I. Utkin (Eds.). Special issue: Sliding mode control in industrial applications. IEEE Trans. Ind. Electron., 55(11), 2008.

[16] J. Bentsman and Y. Orlov. Reduced spatial order model reference adaptive control of spatially varying distributed parameter systems of parabolic and hyperbolic types. Int. J. Adapt. Control Signal Process., 15:679-696, 2001.

[17] M. Bohm, M.A. Demetriou, S. Reich, and I.G. Rosen. Model reference adaptive control of distributed parameter systems. SIAM J. Control Optim., 36 (1):33-81, 1998.

[18] S. Boyd, E. Feron, L. El-Ghaoui, and V. Balakrishnan. Linear matrix inequality in system and control theory. SIAM, Philadelphia, 1994.

[19] C. Brezinski. Computational aspects of linear control. Kluwer, Dordrecht, 2002. 
[20] P.D. Christofides. Robust control of parabolic PDE systems. Chem. Eng. Sci., 16:2949-2965, 1998.

[21] P.D. Christofides. Nonlinear and robust control of PDE systems. Birkhäuser, New York, 2000.

[22] P.D. Christofides and P. Daoutidis. Feedback control of hyperbolic PDE systems. AIChE Journal, 42(11):3063-3086, 1996.

[23] P.D. Christofides and P. Daoutidis. Nonlinear control of diffusion-convection-reaction processes. Computers \& Chemical Engineering, 20(2):S1071 - S1076, 1996.

[24] R.F. Curtain, M.A. Demetriou, and I. Kazufumi. Adaptive compensators for perturbed positive real infinite-dimensional systems. Int. J. Appl. Math. Comput. Sci., 13(4):441-452, 2003.

[25] R.F. Curtain and H. Zwart. An Introduction to Infinite-Dimensional Linear Systems Theory. SpringerVerlag, New York, 1995.

[26] S. Dubljevic, P.D. Christofides, and I.G. Kevrekidis. Distributed nonlinear control of diffusionreaction processes. Int. J. Robust Nonlinear Control, 14:133-156, 2004.

[27] C. Edwards and S.K. Spurgen. Sliding mode control: Theory and applications. Taylor and Francis Ltd., London, 1998.

[28] N.H. El-Farra, A. Armaou, and P.D. Christofides. Analysis and control of parabolic PDE systems with input constraints. Automatica, 39(4):715-725, 2003.

[29] A.F. Filippov. Differential equations with discontinuous righthand sides. Kluwer, Dordrecht, 1988.

[30] L.M. Fridman (Ed.). Special issue: Dedicated to Vadim Utkin on the occasion of his 65th birthday. Int. J. Control, 76(9-10), 2003.

[31] B. Friedland. Control system design: An introduction to state-space methods. McGraw-Hill, New York, 1986.

[32] N. Fuji. Feedback stabilization of distributed parameter systems by a functional observer. SIAM J. Control Optim., 18:108121, 1980. 
[33] J.P. Gauthier, H. Hammouri, and S. Othman. A simple observer for nonlinear systems - Application to bioreactors. IEEE Trans. Autom. Cont., 37(6):875-880, 1992.

[34] L.T. Gruyitch. Tracking control of linear systems. CRC Press, Boca Raton, 2013.

[35] E.M. Hanczyc and A. Palazoglu. Sliding mode control of nonlinear distributed parameter chemical processes. Ind. Eng. Chem. Res., 34(2):557-566, 1995.

[36] M. Izadi and S. Dubljevic. Order-reduction of parabolic PDEs with time-varying domain using empirical eigenfunctions. AIChE J., 59(11):4142-4150, 2013.

[37] I. Karafyllis and C. Kravaris. Robust output feedback stabilization and nonlinear observer design. Sys. \& Contr. Lett., 54:925-938, 2005.

[38] I. Karafyllis and C. Kravaris. Global exponential observers for two classes of nonlinear systems. Sys. \& Contr. Lett., 61(7):797-806, 2012.

[39] N. Kazantzis and C. Kravaris. Nonlinear observer design using Lyapunov's auxiliary theorem. Sys. \& Contr. Lett., 34:241-247, 1998.

[40] H. Keller. Non-linear observer design by transformation into a generalized observer canonical form. Int. J. Contr., 46(6):1915-1930, 1987.

[41] H.K. Khalil. Nonlinear systems. Prentice-Hall, New Jersey, 2002.

[42] M. Krstic and A. Smyshlyaev. Adaptive boundary control for unstable parabolic PDEs - Part I: Lyapunov design. IEEE Trans. Automatic Control, 53:1575-1591, 2008.

[43] L. Lao, M. Ellis, and P.D. Christofides. Economic model predictive control of transport-reaction processes. Ind. Eng. Chem. Res., 53 (18):7382-7396, 2014.

[44] Y.H. Lin and R.A. Adomaitis. Simulation and model reduction methods for an RF plasma glow discharge. J. Comput. Phys., 171(2):731-752, 2001. 
[45] L. Liu, B. Huang, and S. Dubljevic. Model predictive control of axial dispersion chemical reactor. $J$. Process Contr., 24(11):1671-1690, 2014.

[46] C. Lobry, T. Sari, and S. Touhami. On Tykhonov's theorem for convergence of solutions of slow and fast systems. Electronic Journal of Differential Equations (EJDE), 1998(19):1-22, 1998.

[47] P. Lu, J.H. Edgar, O.J. Glembocki, P.B. Klein, E.R. Glaser, J. Perrin, and J. Chaudhuri. High-speed homoepitaxy of SiC from methyltrichlorosilane by chemical vapor deposition. J. Cryst. Growth, 285(4):506-513, 2005.

[48] W. Marquardt. Traveling waves in chemical processes. Int. Chem. Engng., 4:585-606, 1990.

[49] H. Michalska and D.Q. Mayne. Moving horizon observers and observer-based control. IEEE Trans. Autom. Cont., 40(6):995-1006, 1995.

[50] E.A. Misawa and V.I. Utkin (Eds.). Special issue: Variable structure systems. J. Dyn. Syst. Meas. Control, 122, 2000.

[51] J. Ng and S. Dubljevic. Optimal boundary control of a diffusion-convection-reaction PDE model with time-dependent spatial domain: Czochralski crystal growth process. Chem. Eng. Sci., 67(1):111-119, 2012.

[52] R. Penrose. A generalized inverse for matrices. Proceedings of the Cambridge Philosophical Society, 51:406-413, 1955.

[53] W.H. Ray. Advanced process control. McGraw-Hill, Stoneham, 1981.

[54] C. Scherer, P. Gahinet, and M. Chilali. Multiobjective output-feedback control via LMI optimization. IEEE Trans. Automatic Control, 42:896-911, 1997.

[55] L. Sirovich. Turbulence and the dynamics of coherent structures: Parts I, II and III. Quarterly of Applied Mathematics, XLV:561-590, 1987.

[56] J.J.E. Slotine and W. Li. Applied nonlinear control. Prentice-Hall, New Jersey, 1991. 
[57] A. Smyshlyaev and M. Krstic. Backstepping observers for a class of parabolic PDEs. Sys. \& Contr. Lett., 54(7):613-625, 2005.

[58] A. Smyshlyaev and M. Krstic. Adaptive control of parabolic PDEs. Princeton Univ. Press, Princeton, NJ, 2010.

[59] M. Soroush. Nonlinear state-observer design with application to reactors. Chem. Eng. Sci., 52:387404, 1997.

[60] G. Teschl. Ordinary differential equations and dynamical systems: Graduate Studies in Mathematics. Amer. Math. Soc., Providence, 2012.

[61] F.E. Thau. Observing state of nonlinear dynamic-systems. Int. J. Contr., 17(3):471-479, 1995.

[62] A. Theodoropoulou, R.A. Adomaitis, and E. Zafiriou. Model reduction for optimization of rapid thermal chemical vapor deposition systems. IEEE Trans. Semicond. Manuf., 11(1):85-98, 1998.

[63] V.I. Utkin. Sliding modes in control and optimization. Springer-Verlag, Berlin, 1992.

[64] A. Varshney, S. Pitchaiah, and A. Armaou. Feedback control of dissipative distributed parameter systems using adaptive model reduction. AIChE J., 55:906-918, 2009.

[65] C.Z. Xu, P. Ligarius, and J.P. Gauthier. An observer for infinite-dimensional dissipative bilinear systems. Computers Math. Applic., 29(7):13-21, 1995.

[66] Y. Yang and S. Dubljevic. Linear matrix inequalities (LMIs) observer and controller design synthesis for parabolic PDE. European Journal of Control, 20(5):227-236, 2014.

[67] A.S. Zinober. Deterministic control of uncertain systems. Peter Peregrinus Ltd., London, 1990. 
Table 1: Dominant eigenvalues

\begin{tabular}{ccccccc}
\hline \hline Eigenvalues & $i=1$ & $i=2$ & $i=3$ & $i=4$ & $i=5$ & $i=6$ \\
\hline$\lambda_{i}$ & -1.94 & -4.80 & -10.97 & -20.93 & -34.78 & -52.56 \\
\hline
\end{tabular}




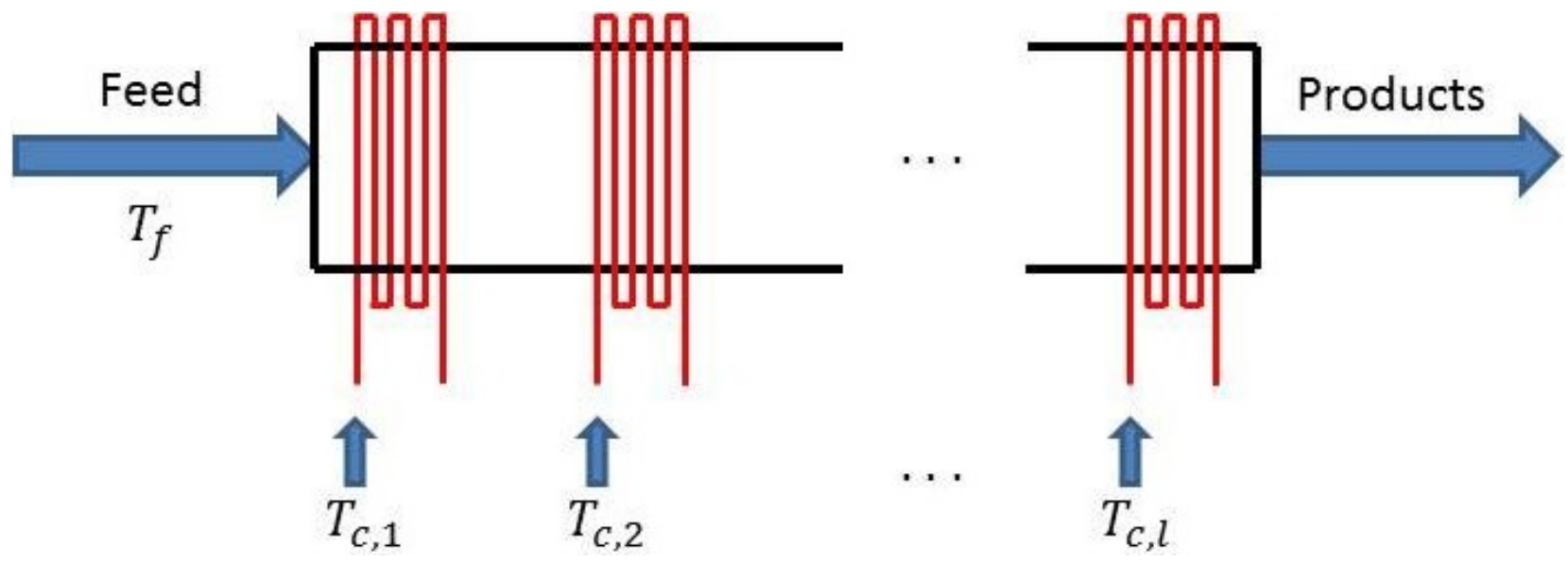

Figure 1: Tubular flow reactor with $l$ independent cooling jackets. 

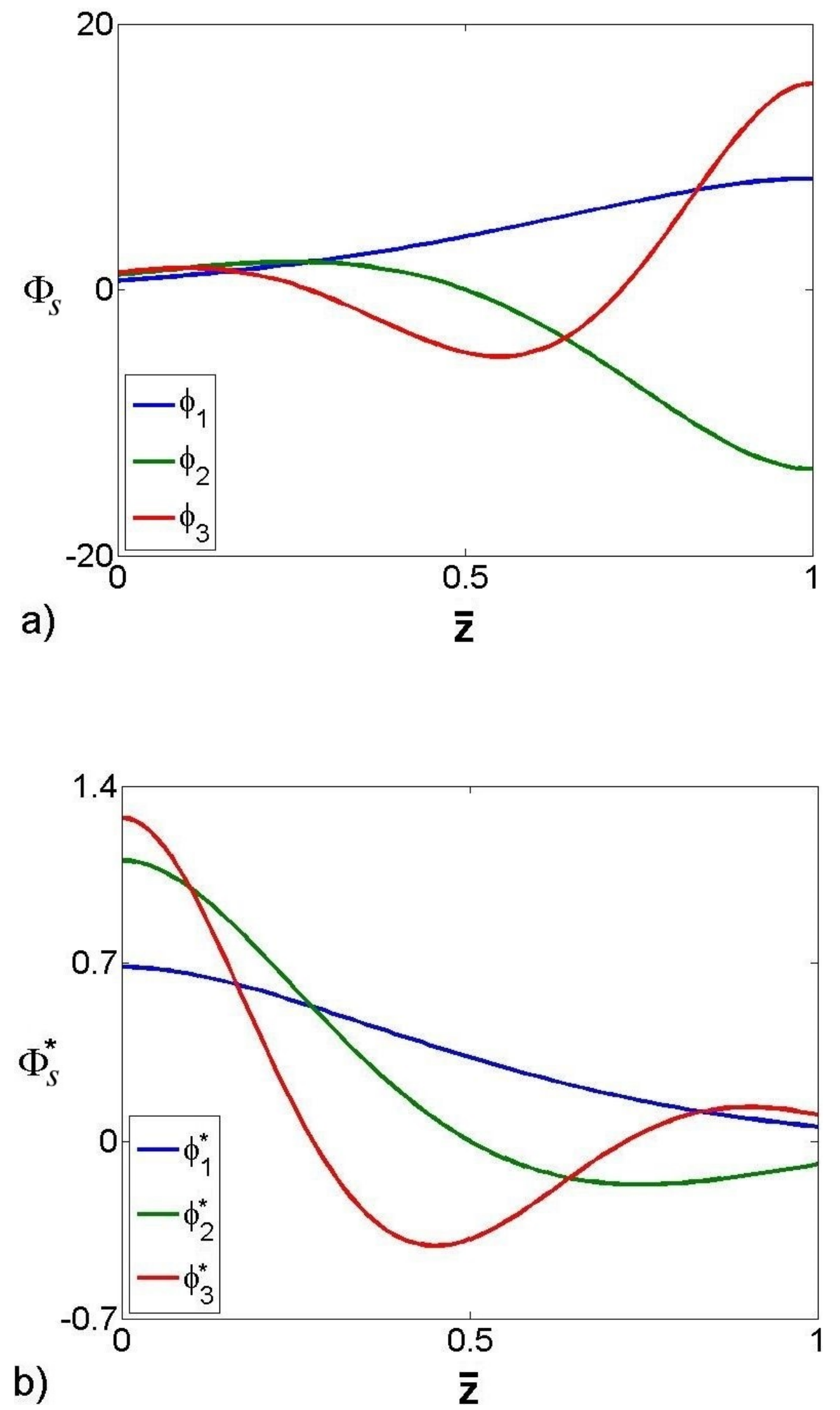

Figure 2: (a) Temperature dominant eigenfunctions and (b) their adjoints. 

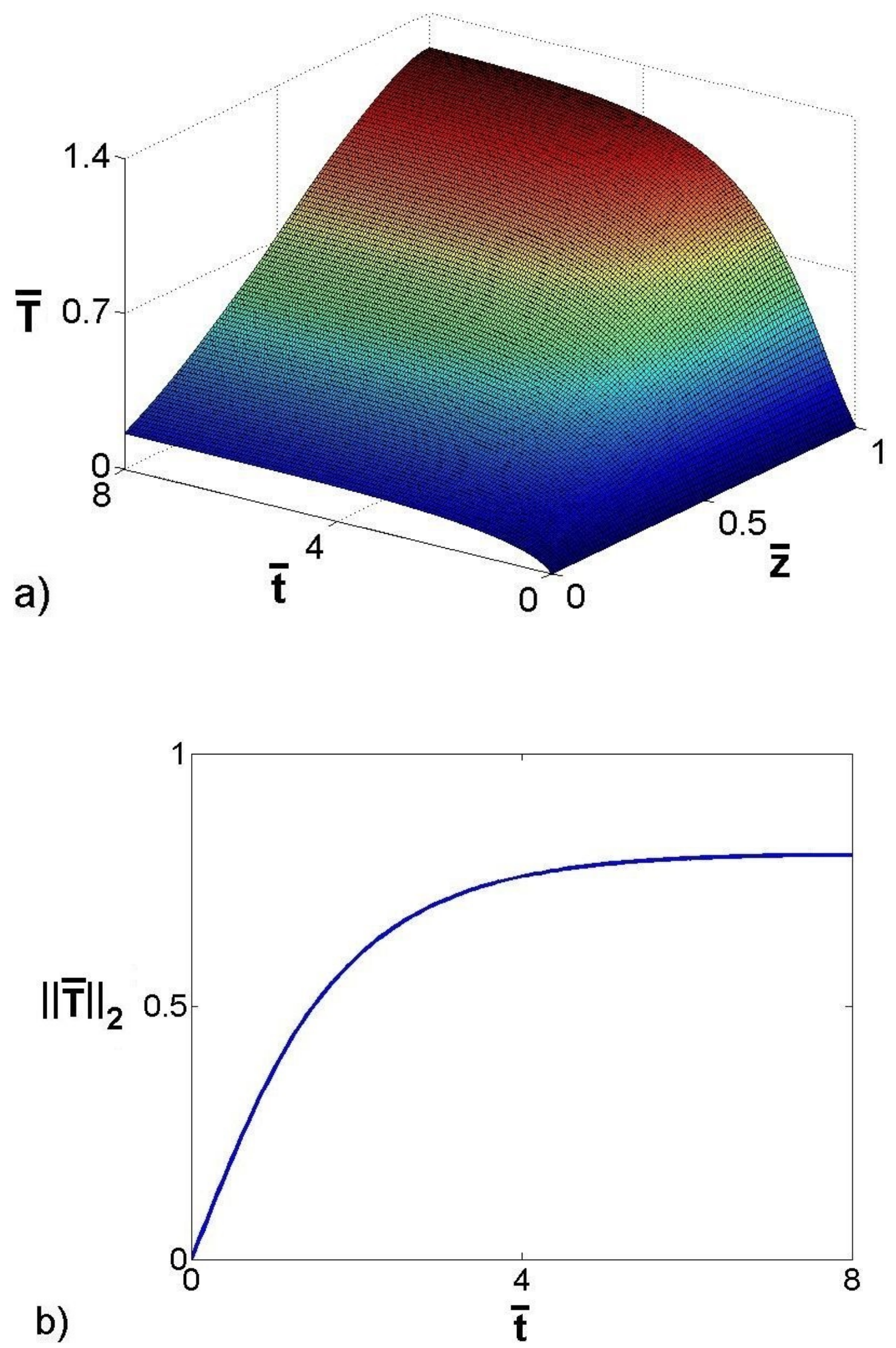

Figure 3: Open-loop (a) spatiotemporal profile of the dimensionless temperature and (b) temporal profile of temperature $L_{2}$ norm. 

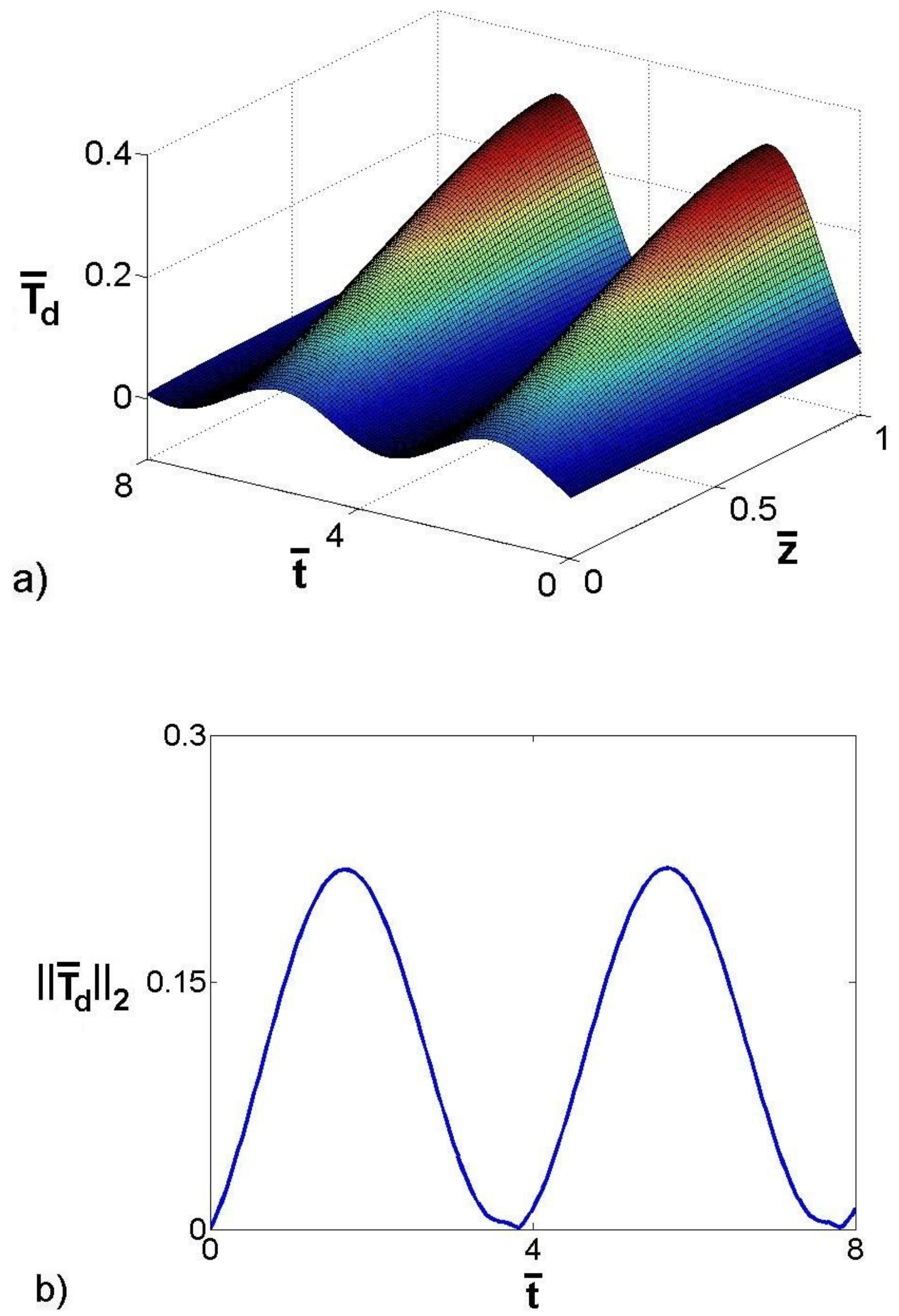

Figure 4: (a) Spatiotemporal profile of the desired dimensionless temperature and (b) temporal profile of its $L_{2}$-norm. 

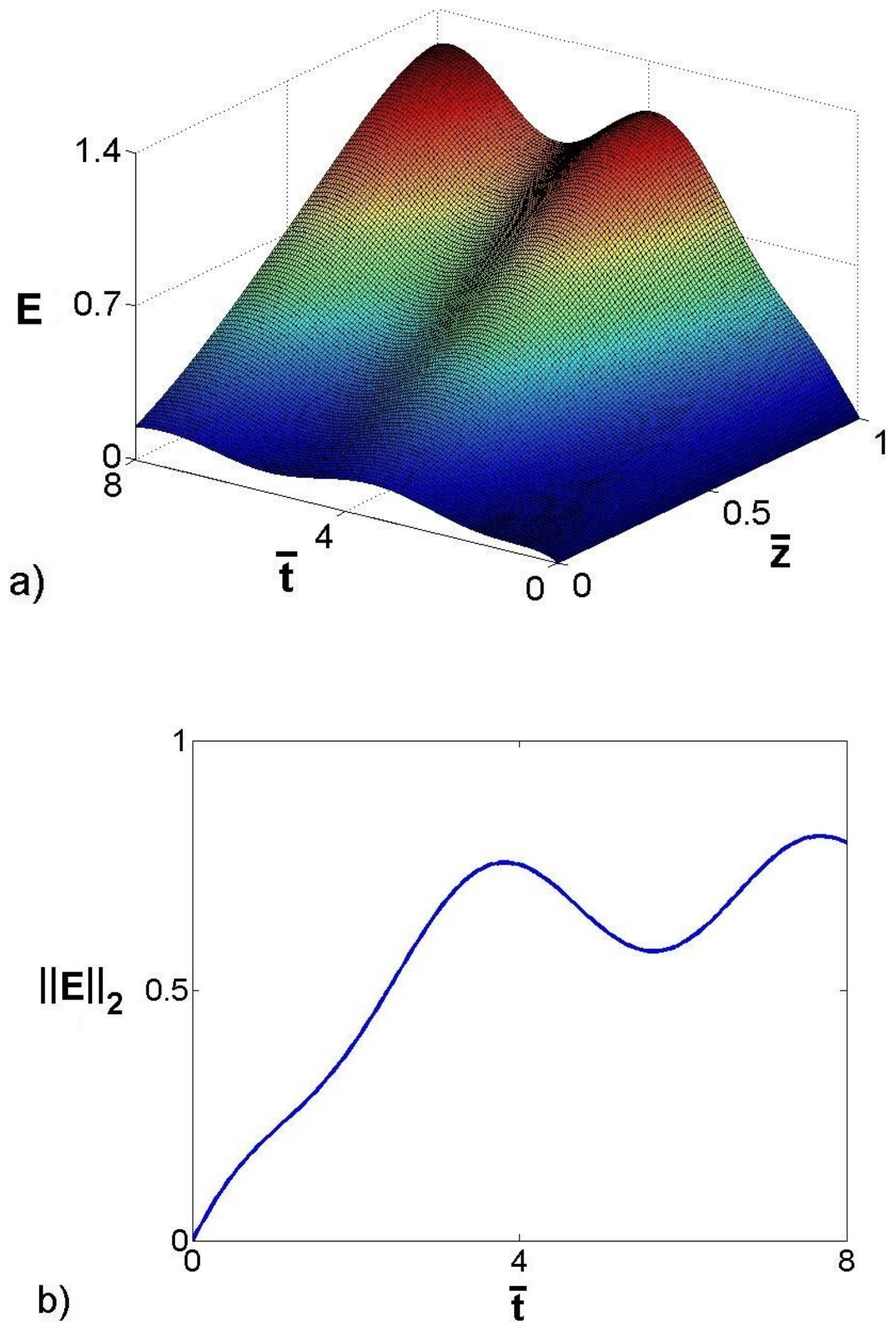

Figure 5: Open-loop (a) spatiotemporal profile of the shaping error and (b) temporal profile of its $L_{2}$-norm. 

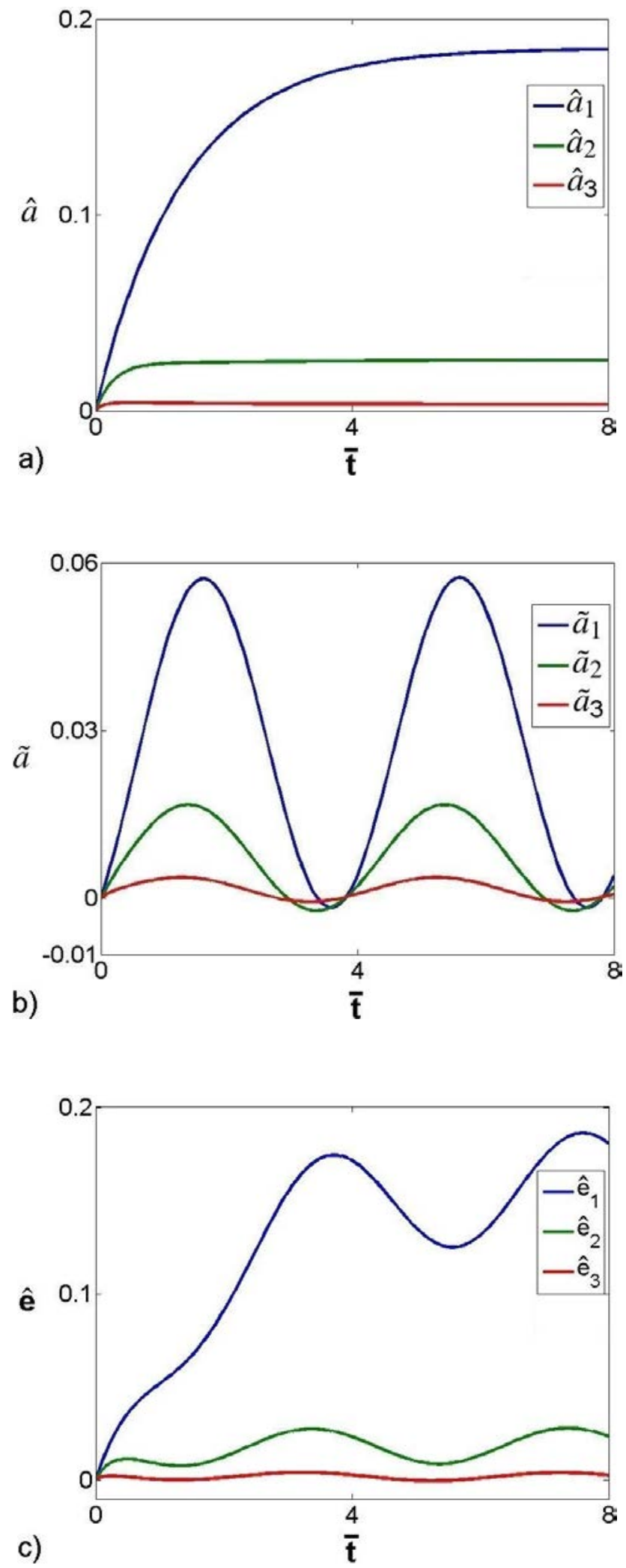

Figure 6: Temporal profiles of (a) dominant eigenmodes of the system, (b) desired dominant eigenmodes and (c) modal errors during the open-loop period. 

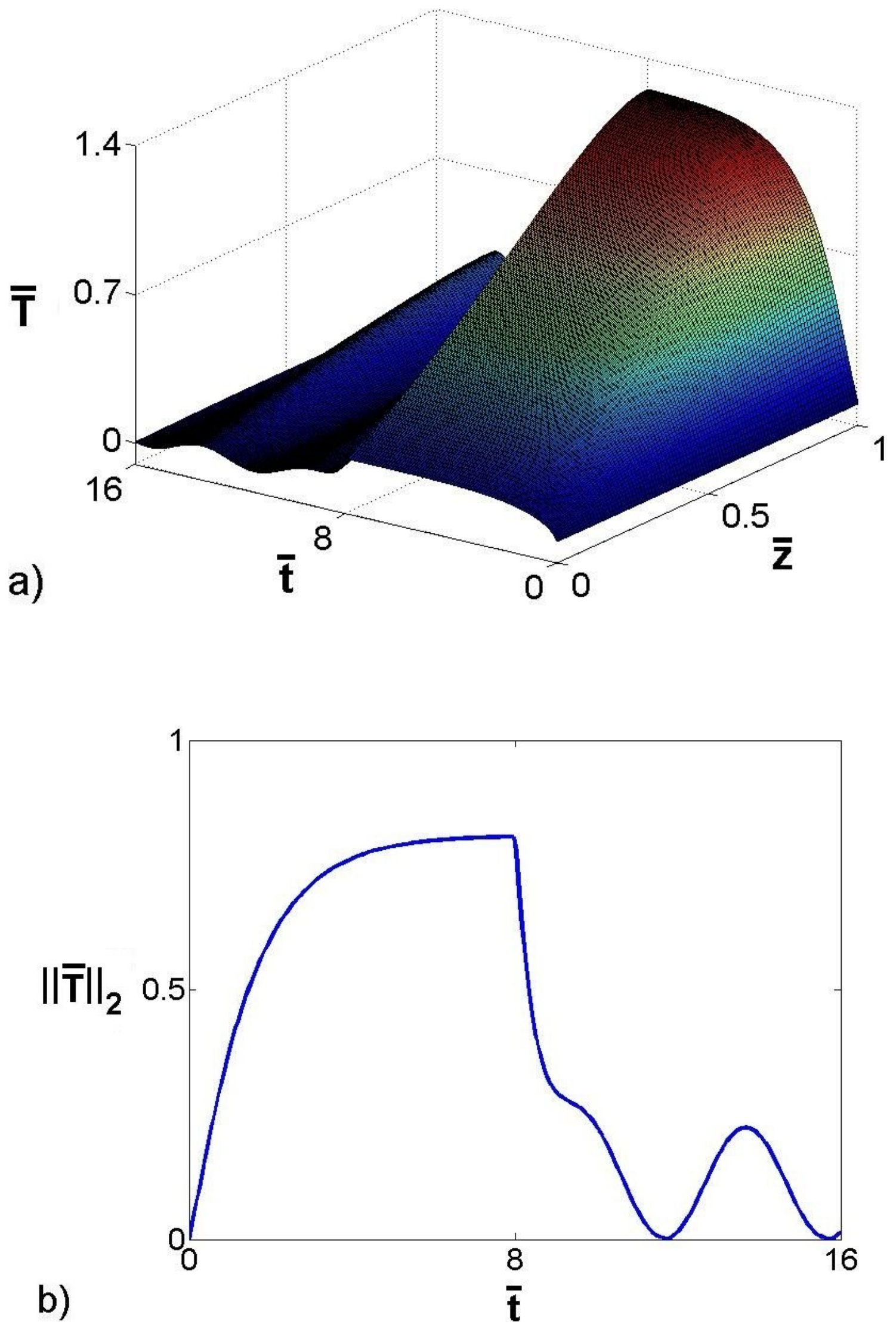

Figure 7: (a) Spatiotemporal profile of the dimensionless temperature and (b) temporal profile of its $L_{2}$-norm for the entire process operation. 

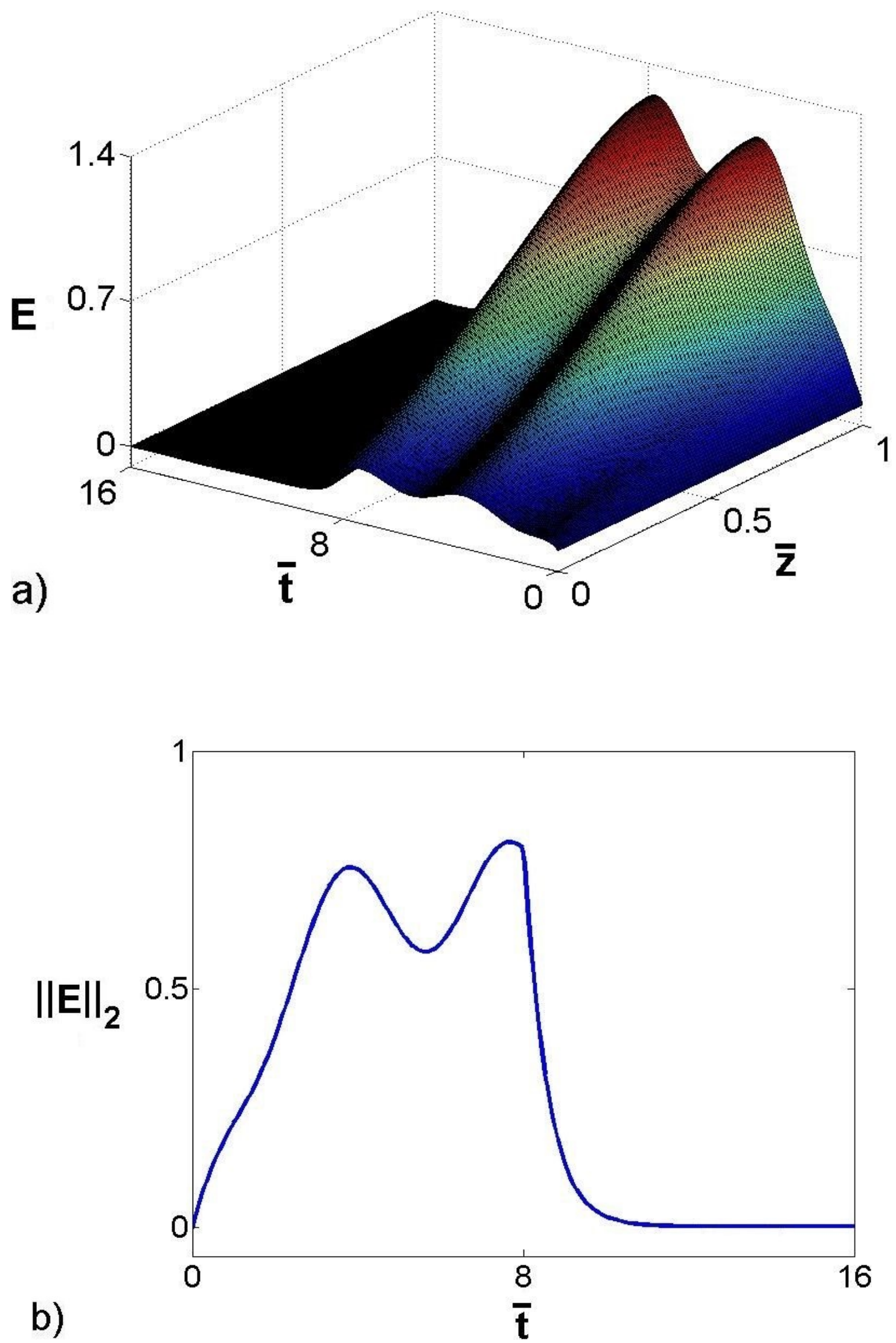

Figure 8: (a) Spatiotemporal profile of the shaping error and (b) temporal profile of its $L_{2}$-norm for the entire process operation. 


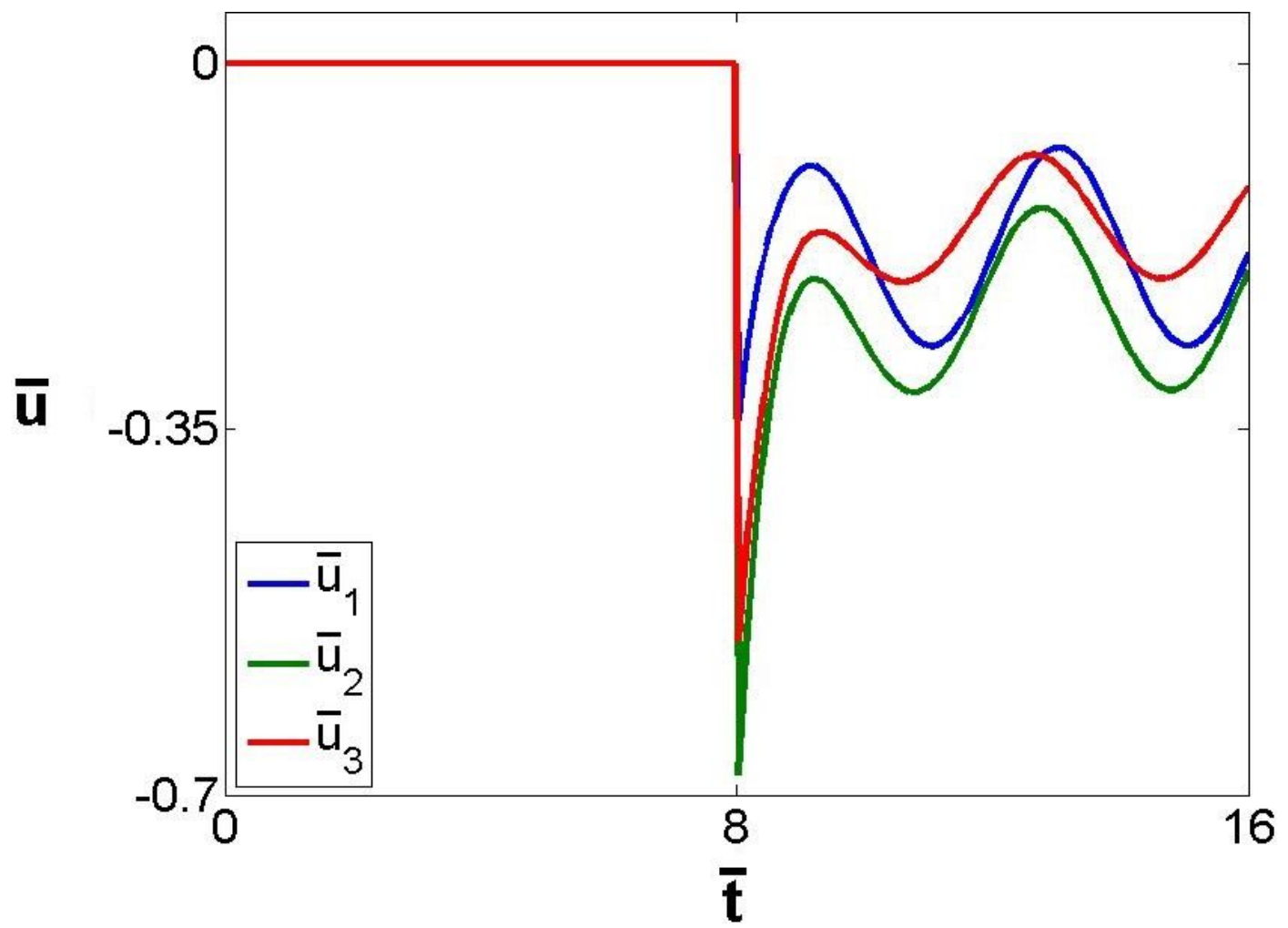

Figure 9: Required control actions for the entire process operation. The zero in $[0,8]$ denotes the open-loop period. 

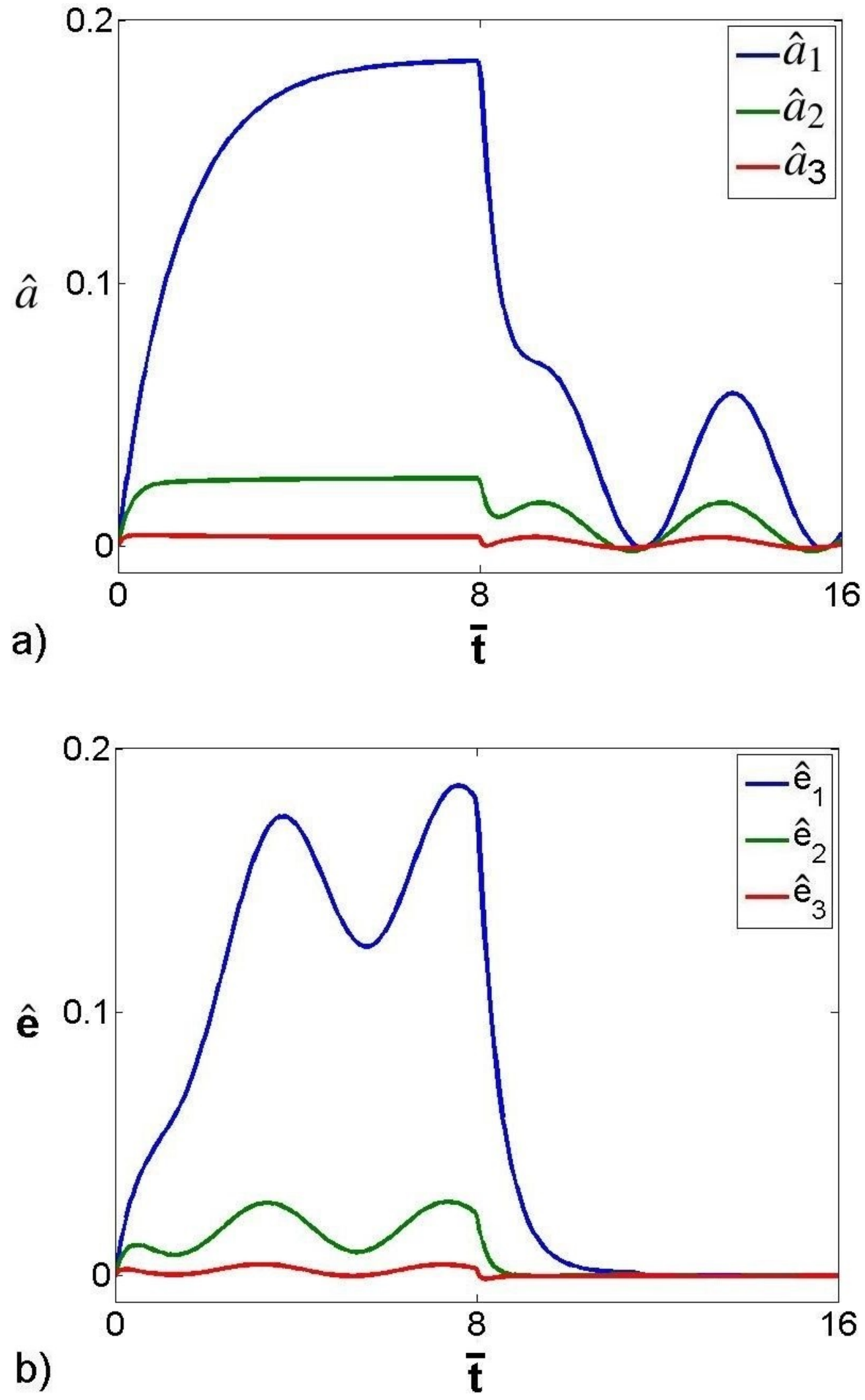

Figure 10: Temporal profiles of (a) dominant eigenmodes of the system and (b) modal errors for the entire process operation. 